\title{
A Taxonomy of Argumentation Models used for Knowledge Representation
}

\author{
By \\ Jamal Bentahar* $^{*}$ Bernard Moulin ${ }^{+\dagger}$, Micheline Bélanger ${ }^{\ddagger}$
}

* Concordia Institute for Information Systems Engineering, Concordia University, 1425 René Lévesque Blvd. West, CB-410-10, Montreal, Quebec, H3G 1T7,Canada. Phone: (514) 848-2424 ext: 5382

Fax: (514) 848-3171

${ }^{+}$Department of Computer Science and Software Engineering, Laval University, Ste

Foy, G1K 7P4, Canada.

Phone: (418) 656-5580

Fax: (418) 656-2324

${ }^{\dagger}$ Research Center on Geomatics, Laval University, Canada.

‡ Defence Research and Development Canada, Valcartier, Quebec, G3J 1X5, Canada.

E-mail: bentahar@ciise.concordia.ca, bernard.moulin@ift.ulaval.ca, micheline.belanger@drdc-rddc.gc.ca

\begin{abstract}
Understanding argumentation and its role in human reasoning has been a continuous subject of investigation for scholars from the ancient Greek philosophers to current researchers in philosophy, logic and artificial intelligence. In recent years, argumentation models have been used in different areas such as knowledge representation, explanation, proof elaboration, commonsense reasoning, logic programming, legal reasoning, decision making, and negotiation. However, these models address quite specific needs and there is need for a conceptual framework that would organize and compare existing argumentation-based models and methods. Such a framework would be very useful
\end{abstract}


especially for researchers and practitioners who want to select appropriate argumentation models or techniques to be incorporated in new software systems with argumentation capabilities. In this paper, we propose such a conceptual framework, based on taxonomy of the most important argumentation models, approaches and systems found in the literature. This framework highlights the similarities and differences between these argumentation models. As an illustration of the practical use of this framework, we

present a case study which shows how we used this framework to select and enrich an argumentation model in a knowledge acquisition project which aimed at representing argumentative knowledge contained in texts critiquing military courses of action.

Key Words: Argumentation Models, Argumentation Theory, Courses of Action, Knowledge Representation.

\section{Introduction}

The issue of understanding argumentation and its role in human reasoning has been addressed by many researchers in various fields such as philosophy, logic, artificial intelligence, and software engineering (Rahwan and McBurney, 2007). A well-known example of an argument from artificial intelligence is Tweety flies because Tweety is a bird, which can be counter-argued by the argument But Tweety is different, so perhaps Tweety does not fly. In epistemology, the standard example is: This looks red, therefore it is red, which can be counter-argued by But the ambient light is red, so perhaps it is not red. In law, the famous example is $A$ contract exists because there was offer, acceptance, memorandum, and consideration, which can be counter-argued by But one of the parties to the contract is incompetent, so there is no contract. These are one-step arguments in a two-party dialogue. Arguments can also be chained and dialogues can run deeper.

In recent years, argumentation has been used in different areas such as knowledge representation, explanation, proof elaboration, commonsense reasoning, logic programming, legal reasoning, decision making, and negotiation (Prakken and Sartor, 1997; Kakas et al., 1999; Amgoud and Cayrol, 2000; Besnard and Hunter, 2001; Prakken, and Vreeswijk, 2002; Rahwan et al., 2004; Atkinson et al., 2005; Bentahar, 2005; 
Bentahar, 2010). Argumentation theory has also been applied in the design of intelligent systems in several ways over the last decade (Moulin et al., 2002). Some examples of systems implementation (Verheij, 1998) are: IACAS (Vreeswijk, 1995), Room5 (Loui et al., 1997), Dialaw (Lodder, 1998), Argue! (Verheij, 1998), PROforma (Fox and Das, 2000), React (Glasspool et al., 2003), Parma (Greenwood et al., 2003), Hermes (Karakapilidis, 2001) and HYPO family (Ashley and Rissland, 2003; Brueninghaus and Ashley, 2003).

The argumentation models developed during the past two decades addressed specific needs for various domains such as legal reasoning, multi-agent systems, agent communication and natural argumentation (Reed and Norman, 2003; Bench-Capon and Prakken, 2006). Focusing mostly on specific domain needs and specific classes of formalisms (e.g. logic-based formalisms), researchers have not really developed a global view of existing argumentation models and methods. This lack of global view is a real problem when an argumentation model needs to be selected for the development of new software systems with intelligent components such as decision-making, explanation, or evaluation of some situations. Furthermore, the need of such a view for developing a unified architecture for argumentation-based systems has been identified and highlighted in the recent developments of an argumentation interchange format for multi-agent systems aiming to propose a unified language for argument representation (Willmott et al., 2006; Chesnevar et al., 2006; Rahwan et al., 2007). To alleviate this deficiency, we propose in this paper a taxonomy of the most important argumentation models, approaches and systems found in the literature. Moreover, we propose a conceptual framework as a layered architecture representing a global view that illustrates the links between different classes of argumentation approaches identified in our taxonomy.

Another contribution of this paper is the comparison of the different argumentation models in each category of the proposed taxonomy. Highlighting the similarities and differences between these argumentation models should help researchers and developers to determine which model is the most appropriate for their application domain. In 
addition, the comparison framework of argumentation models that we propose may be used to identify further research areas.

Paper Overview. In Section 2, we introduce the argumentation theory and a conceptual framework representing the different classes of argumentation models as identified in our taxonomy. In Sections 3, 4, and 5, we survey the main argumentation models that have been proposed in the literature. We present this review using our taxonomy: monological models (Section 3), dialogical models (Section 4), and rhetorical models (Section 5). In Section 6, we present a case study, which shows how we used this framework to select and enrich an argumentation model in a knowledge acquisition project, which aimed at representing argumentative knowledge contained in texts critiquing military courses of action. Finally, Section 7 concludes the paper.

\section{A Global View of Argumentation Models}

Arguments can be considered as tentative proofs for propositions (Fox et al., 1993), (Kraus et al., 1995). In formal argumentation, knowledge is expressed in a logical language, with the axioms of the language corresponding to premises according to the underlying domain (Prakken and Vreeswijk, 2002). Theorems in the language correspond to claims in the domain which can be derived from the premises by successive applications of some inference rules. Generally, the premises are inconsistent in the sense that contrary propositions may be derived from them. In this formulation, arguments for propositions, or claims, are the same as proofs in a deductive logic, except that the premises on which these proofs rest are not all known to be true.

The understanding of an argument as a tentative proof and a chain of rules attends to its internal structure. Several models addressing the internal structure of arguments have been developed, for example (Toulmin, 1958; Farley and Freeman, 1995; Reed and Walton, 2003). These models stress the link between the different components of an argument and how a conclusion is related to a set of premises. They mainly consider the relationships that can exist between the different components of an argument in a 
monological structure. For this reason, we call the models belonging to this category: monological models.

A second strand of research in artificial intelligence has emphasized the relationships existing between arguments, some times considered as abstract entities and ignoring their internal structures. Because they highlight the structure of arguments as presented in a dialogical framework, the models belonging to this category are called dialogical models. Several dialogical models have been proposed in the literature, for example (Atkinson et al., 2006; Bentahar et al., 2004a; Bentahar et al., 2004b; Dung, 1995; Hamblin, 1970; MacKenzie, 1979).

In the philosophy of argumentation, several researchers have distinguished the process of argument from the product of argument (Habermas, 1984; O'Keefe, 1977). According to (Johnson, 2000), it is possible to see dialogue logic as having its focus on the process of arguing, whereas informal logic is focused on the product. The process of arguing is related to the dialogical models, whereas producing arguments is a part of monological considerations. Normally, in informal logic, the aim is to identify, analyze or evaluate an argument found in a text. The argument is thought of as a product composed of a set of premises offering support to a conclusion. Monological and dialogical views are consequently strongly connected. First, to identify the argument, and to classify it as an argument, as opposed to some other speech act types like explanations, one has to identify the conclusion as a specific proposition that could be refused or attacked. This determination presupposes a dialogical viewpoint in which there are two players: the proponent supporting the conclusion and the opponent attacking the premises or conclusion (Reed and Walton, 2003).

Dialogical models have enabled argumentation systems to be defined as defeasible reasoning systems. Arguments are thus defeasible, meaning that the argument by itself is not a conclusive reason for the conclusions it brings about. When a rule supporting a conclusion may be defeated by new information, it is said that such reasoning is defeasible (Pollock, 1974). When we chain defeasible reasons to reach a conclusion, we 
have arguments, instead of proofs. In defeasible logic (also called non-monotonic logic), inferences are defeasible because they can be defeated when additional information becomes available. Several non-monotonic reasoning formalisms have been proposed. In these formalisms, conclusions which have been drawn may be later withdrawn, when additional information becomes available. Several logic-based argumentation systems have been proposed to perform this type of reasoning (Pollock, 1994).

Generaly, monological models and dialogical models consider respectively the internal (micro) and external (macro) structure of arguments. Other models, which do not take into account these structures, have been defined, for example by (Olbrechts-Tyteca, 1969; Cabrol-Hatimi, 1999; Grasso, 2002; Pasquier et al., 2006; Gordon et al., 2007). These models, that we call rhetorical models, consider rather the rhetorical structure of arguments (e.g. rhetorical patterns or schemas). They aim at studying the way of using arguments as a means of persuasion. A fundamental characteristic of these models is the fact that they consider the audience's perception of arguments. In fact, very few dialogical models (Bench-Capon, 2003; Bentahar et al., 2007b) have this characteristic, which makes them exhibiting both rhetorical and dialogical features. Rhetorical models deal with arguments, which are both based on the audience's perception of the world, and with evaluative judgments rather than with establishing the truth of a conclusion.

Excepting the model proposed by Bench-Capon (1989), Farley and Freeman's proposal (1995), and Atkinson and her colleagues' model (2006), which combine monological and dialogical structures of arguments, the models belonging to a given category are, as presented in the literature, completely independent from the models belonging to the two other categories. For example, dialogical models do not take into account the microstructure of arguments and the audience's perception of such arguments. However, in order to design and implement intelligent systems with efficient argumentative capabilities, micro, macro and rhetorical structures of arguments should be addressed. Arguments should be efficiently built at the internal level in terms of the relations between premises and conclusions and at the external level in terms of the relations between the other arguments that can be produced previously. In addition, these 
arguments should be produced by taking into account the audience's perception because in real life applications, arguments are produced to reach some predetermined goals depending on the participating agents' believes. Monological, dialogical and rhetorical models are then complementary. Indeed, if the micro-structure of arguments is not considered, it will be difficult to produce arguments able to convince the audience, and if the rhetorical structure is not taken into account, the relations between arguments in the macro-structure cannot be efficient enough. Figure 1 illustrates our conceptual framework of these model classes with the main characteristics of each class. These classes can be structured in a layered framework which has the advantage of illustrating the abstract level of arguments when developing argumentative software systems. The micro structure should be addressed at the first level. Once the argument is built, it should be related to the rest of arguments by argumentative relations (macro structure). When the macro-structure is conceived, the audience's set of beliefs should be considered to produce convincing arguments (rhetorical structure).

\section{Monological Models}

While dialogical models and rhetorical models of argumentation highlight the process of argumentation in a dialogue structure, monological models emphasize the structure of the argument itself. What is important in these models is not the relationship that can exist between arguments, but the relationships between the different components of a given argument. In this section, before summarizing the main research work done in monological models, we provide a list of evaluation criteria we use to assess the argumentation frameworks in this category of argumentation models.

\section{Evaluation criteria for monological models}

C1: Definition of the argument structure: specification of the different components of an argument.

C2: Specification of combining the argument components: explanation of how the components can be combined when forming and building arguments within an argumentation process. 
C3: Clarity and relevance of the theoretical foundations: consideration of the foundations used in the definition of arguments and their structures.

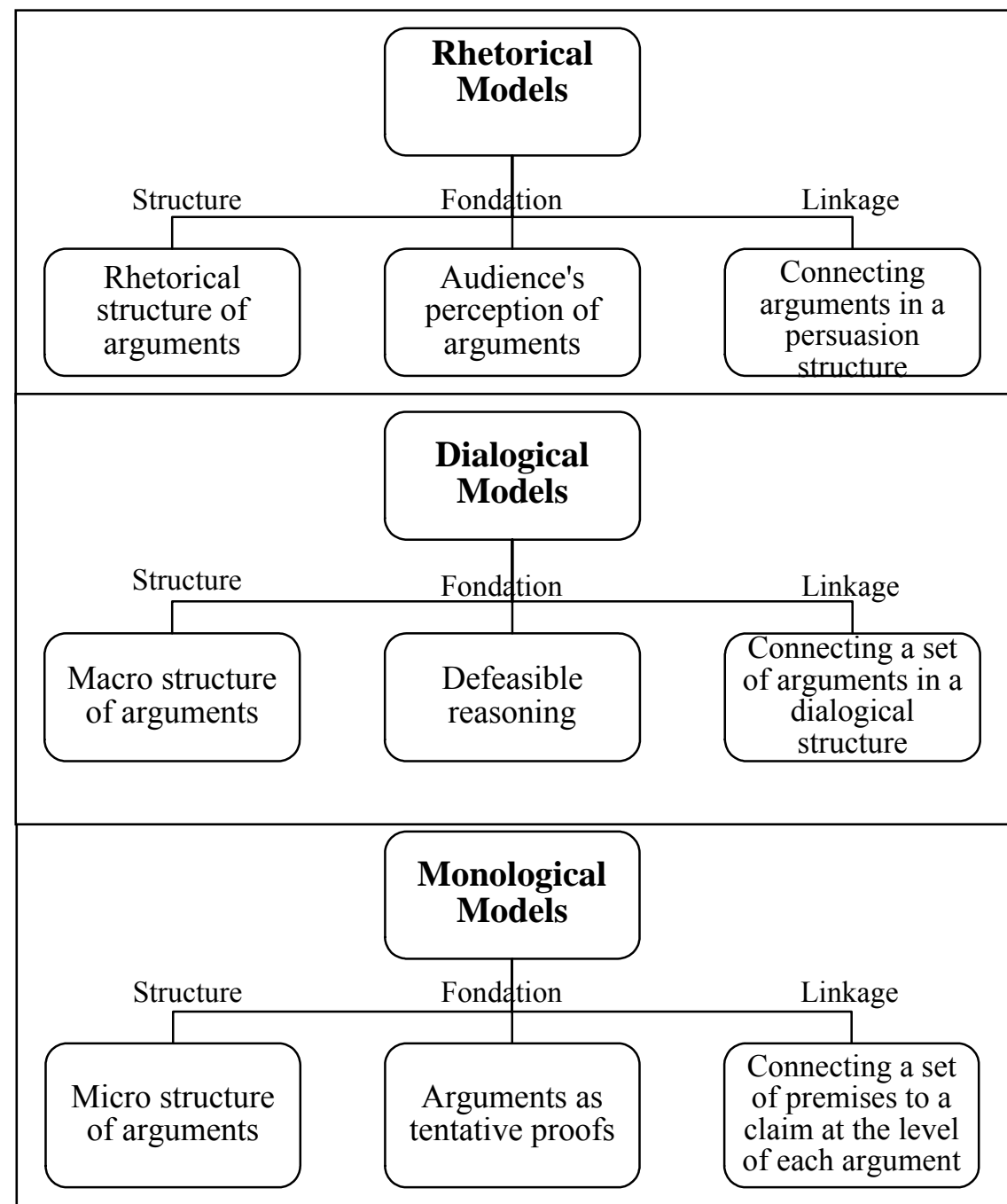

Figure 1. A conceptual framework of the three categories of argumentation models

C4: Applicability of the model: definition of the domains in which the model can be applied along with its purposes, particularly in terms o knowledge representation and knowledge elicitation.

C5: Modeling the inference mechanism: explanation of how a conclusion is inferred from a set of premises. 
C6: Consideration of the participants in the argumentation process: specification of how the participants can use the model and how they are modeled, for example in terms of specifying their knowledge bases.

C7: Specification of the acceptability criteria: explanation of how to decide about the acceptability of an argument.

In the rest of the paper, when a criterion $C i(i \in \mathbb{N})$ is satisfied, it will be denoted $(+C i)$; otherwise, it will be denoted (- $\mathrm{C} i$ ). Some criteria are partially satisfied and this case will be denoted by $( \pm \mathrm{C} i)$.

\subsection{Toulmin's Model and its Extensions}

In a logical proof, we have a set of premises and a conclusion which is said to follow from them. Many argumentation systems make no distinction between their premises. In contrast, in arguments expressed in natural language we can typically observe premises playing different roles. By identifying these roles, we can present the arguments in a more readily understandable fashion, and also identify the various ways in which the argument may be accepted or attacked. Structuring the argument in such a way produces what is commonly called an argument scheme. Analyzing practical reasoning in terms of argument schemes produces taxonomy of arguments, which may provide useful guidance to implement argumentation systems, analogous to the guidance provided by domain ontologies for building knowledge-based systems (Mommers, 2002). One argument scheme that has been widely used in artificial intelligence and law was proposed a long time ago by Toulmin (1958).

In the domain of philosophy of law, Toulmin (1958) introduced a conceptual model of argumentation. He considered a diagrammatic representation for legal arguments, in which six parts are distinguished:

1. Claim (C). An assertion or a conclusion presented to the audience and which has potentially a controversial nature (it might not meet the audience's initial beliefs).

2. Data $(D)$. Statements specifying facts or previously established beliefs related to a situation about which the claim is made.

3. Warrant $(W)$. Statement, which justifies the inference of the claim from the data. 
4. Backing $(B)$. Set of information, which assures the trustworthiness of a warrant. A backing is invoked when the warrant is challenged. The backing is the ground underlying the reason.

5. Qualifier $(Q)$. A statement that expresses the degree of certainty associated to the claim.

6. Rebuttal $(R)$. A statement presenting a situation in which the claim might be defeated.

Counterarguments are also arguments that may attack any of the first four elements (Claim, Data, warrant and Backing). A disputation can be visualized by chaining diagrams of arguments.

This structure may be represented using typical natural language markers:

Given $D$ (and Since $W$ ), Therefore $C$, unless $R$.

$W$ Because $B$.

Figure 2 illustrates an example of Toulmin's model. This argument claims that Harry is a British citizen (Claim) because he was born in Bermuda (Data). This claim is presumably true since people born in Bermuda are generally British citizens (Warrant) because there are statutes and other legislation substantiating this rule (Backing). However, there are exceptions to this rule, such as when a person born in Bermuda has parents of another nationality or if this person becomes a naturalized American citizen (Rebuttal). 
Harry was born in Bermuda

People born in Bermuda are generally British citizens

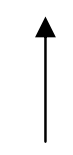

Presumably (Qualifier), Harry is

a British citizen (Claim)

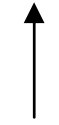

Harry's parents have another nationality or

Harry becomes a naturalized American citizen (Rebuttal)

there are statutes and other legislation substantiating that people born in Bermuda are generally British citizens (Backing)

Figure 2. An illustration of Toulmin's argument structure

Ye (1995) indicated that Toulmin's model is significant in that it highlights the discrete response steps that an expert system explanation facility should follow in order to answer a user's queries in a convincing way. For example, let us consider the typical format of a rule used in an expert system:

IF Premise (certainty factor $_{y}$ ), THEN Conclusion $z$.

This structure obviously corresponds to the schema (subscript variables represent the correspondence between the elements of these structures):

GIVEN Data $x$,THEREFORE (Qualifier (Claim $_{z}$.

Certain rules might include the equivalent of a rebuttal as for example:

IF Premise $_{x}$ AND NOT Premise (certainty factor $_{z}$ ), THEN Conclusion . $_{\text {. }}$

This structure corresponds to the schema:

GIVEN Data , THEREFORE Qualifier $_{z}$ Claim $_{w}$, UNLESS Rebuttal.

Although Toulmin's model has been used in several research works on argumentation, it is possible for an argument to lack one or more of the components of Toulmin's argument structure. Indeed, weaker arguments often have significant holes as for example, in the data supporting the claim or in the backing supporting the warrant or in considering rebuttals. 
Bench-Capon (1989) introduced an additional component to Toulmin's structure: the presupposition component which represents assumptions that are necessary for the argument but are not the object of dispute and remain outside the core of the argument. Bench-Capon used this modified form of Toulmin's schema to represent argumentative knowledge and as a basis for the definition of a dialogue abstract machine intended to implement a Toulmin dialogue game. Claim (used to assert that a proposition is true), Why (seeks the data for a claim), Ok (accepts a claim) and Presupposing (seeks any presuppositions on which a claim is based) are examples of moves used in Toulmin dialogue game. This dialogue game uses some of the concepts of MacKenzie's game (MacKenzie, 1979), including the idea of a commitment store which records the commitments of the participants, but within a richer framework of rules. The modified form of Toulmin's schema is used to facilitate construction of textual arguments from the results of the dialogue. We can consider the Bench-Capon's extension as a link between monological and dialogical models. This model has been recently enriched by Atkinson et al. (2006). In this paper, the authors present a protocol based on a detailed argument schema for reasoning and arguing about arguments that are associated to actions.

Farley and Freeman (1995) extended the warrant component in order to develop a model of dialectical reasoning. Two types of warrants: wtypel and wtype 2 are distinguished. The wtypel warrant classifies the relationship between assertion and data as explanatory or sign. Causal link are examples of explanatory warrants because they explain an assertion given data. A sign relationship represents a link of correlation between data and assertion. The wtype 2 warrant represents the strength with which the assertion can be drawn from data. The authors distinguished default type warrants which represent default relationships, evidential warrants which are less certain and sufficient warrants which are certain and typically stem from definitions. In (Freeman, 1991) Freeman identified four main argument structures: convergent arguments, linked arguments, arguments in sequence and divergent arguments. In convergent arguments, several premises independently contribute to a unique conclusion. In linked arguments, several premises contribute together to a unique conclusion. In arguments in sequence, the conclusion of a 
sub-argument is the premise of another argument. Finally, in divergent arguments, a unique premise supports different conclusions.

Stranieri and Zeleznikow (1999) suggested that warrants communicate two distinct meanings: a reason for the relevance of a fact and a rule which, when applied to the fact leads us to infer the claim. On the basis of this distinction, the authors explicitly identified three features that are implicit in Toulmin's formulation: (1) an inference procedure, an algorithm or method used to infer an assertion from data; (2) reasons which explain why a data item is relevant for a claim; (3) reasons that explain why the inference method used is appropriate.

Clark (1991) developed an approach to knowledge representation and problem-solving based on Toulmin's argumentation model (1958) and applied it to the domain of geological risk assessment. He developed the Optimist System which involved the ability to compare different user's opinions, to modify the system's model of users' opinions and to allow the user to express his disagreement with the system's choices. In this system, problem-solving is considered as a cooperative activity based on the interaction of different, possibly conflicting chains of reasoning. The interaction involves an exchange of information between the system and the user, discussing why a particular risk is valid. This early argumentation-based expert system was used by expert geologists, all of whom were able to dispute and correct the system's reasoning to their satisfaction. Adopting the principle of consistency, namely that a rational agent will make similar decisions in similar situations, Clark used precedents as a form of justification for the system's arguments. Precedents were stored in and retrieved from a case-base, allowing a simple form of case-based reasoning.

Toulmin's model and its extensions have the following advantages and limits:

\section{Advantages}

- They take into account the different components of an argument structure and the link between these components $(+\mathrm{C} 1,+\mathrm{C} 2)$. 
- They are based on philosophical and empirical foundations (+ C3).

- They facilitate the construction of textual arguments (+ C4).

- They provide an excellent means for knowledge representation (+ C4).

- They can be used for knowledge elicitation because they consider the structure of arguments and how these arguments can be linked $(+\mathrm{C} 4)$.

- They model the inference rules that are used to infer a conclusion from a set of premises (+ C5).

\section{Limits}

- They are based on an informal description. Consequently, the defeasible rules and the relations between the elements of an argument are sometimes ambiguous. For example, the warrant that supports the inference rule is, in some cases, not clear, because this rule is not clearly defined (- C2, - C5).

- They do not formally specify how the different argument structures can be combined in order to illustrate the dynamics of the argumentation process. However, this is supported as a part of Toulmin's dialogue games $( \pm \mathrm{C} 2)$.

- They only emphasize the structure of the arguments without taking into account the participants and their knowledge bases (- C6).

- The acceptability criteria of the arguments are not specified (- C7).

\subsection{Argumentation Schemes proposed by Reed and Walton}

To model the notions of arguments as product, Reed and Walton proposed the notion of argumentation scheme. Argumentation schemes are the forms of arguments describing the structures of inference. This notion enables the authors to identify and evaluate common types of argumentation in everyday discourse. Such schemes can be used to represent knowledge needed for arguing and explaining. They capture common, stereotypical patterns of reasoning which are non-deductive and non-monotonic. To understand this notion, let us take the following example from (Reed and Walton, 2003). Suppose that Bob and Helen are having a critical discussion on tipping, and that Helen is against tipping. She thinks that tipping is a bad practice that ought to be discontinued. Suppose that in this context, Helen puts forward the following argument: 


\section{Dr. Phil says that tipping lowers self-esteem.}

Dr. Phil is an expert psychologist, so the argument is, at least implicitly, an appeal to expert opinion. It is also, evidently, an instance of argument from consequences. Helen is telling her opponent, Bob, that lowering self-esteem is a bad consequence of an action. Her argument is based on the assumption that since this bad outcome is a consequence of tipping, tipping itself is a bad thing. Thus, Helen's argument is an enthymeme. It is a chain of argumentation that can be reconstructed as follows:

\section{The Self-Esteem Argument}

- Dr. Phil says that tipping lowers self-esteem. Dr. Phil is an expert in psychology, a field that has knowledge about self-esteem.

- Tipping lowers self-esteem.

- Lowering self- esteem is a bad thing.

- Anything that leads to bad consequences is itself bad as a practice.

- Tipping is a bad practice.

In this example, argumentation schemes can be used to fill in the unstated premises and to link them together with other premises and conclusions in a chain of argumentation that represents Helen's line of argument. Walton (1996) identified twenty-five argumentation-schemes. The argumentation scheme that can be used in the case of the example is called argument from expert opinion. It is represented as follows:

Major Premise: Source $E$ is an expert in subject domain $S$ containing proposition $A$.

Minor Premise: $E$ asserts that proposition $A$ (in domain $S$ ) is true (false).

Conclusion: $A$ may plausibly be taken to be true (false).

The scheme lets us reconstruct Helen's argumentation by filling in the implicit premises needed to make her argument fit the requirements of the appeal to expert opinion. To fill in the other missing parts of the argument we can use the scheme for argument from consequences. The authors distinguished positive consequences from negative consequences. This scheme is represented as follows: 
Major Premise: If an argument leads to good (bad) consequences, it should (should not) be brought about.

Minor Premise: If action $A$ is brought about, good (bad) consequences will occur.

Conclusion: Therefore $A$ should (should not) be brought about.

This argumentation scheme can be used to give a reason to support the claim that an action should not be carried out. The reason offered is that bad consequences will occur. In this case, it has been shown how both schemes can be used to help insert missing parts of an argument needed to reconstruct the argumentation in the case of forward chaining.

The self-esteem argument can be diagrammed as illustrated by Figure 3 .

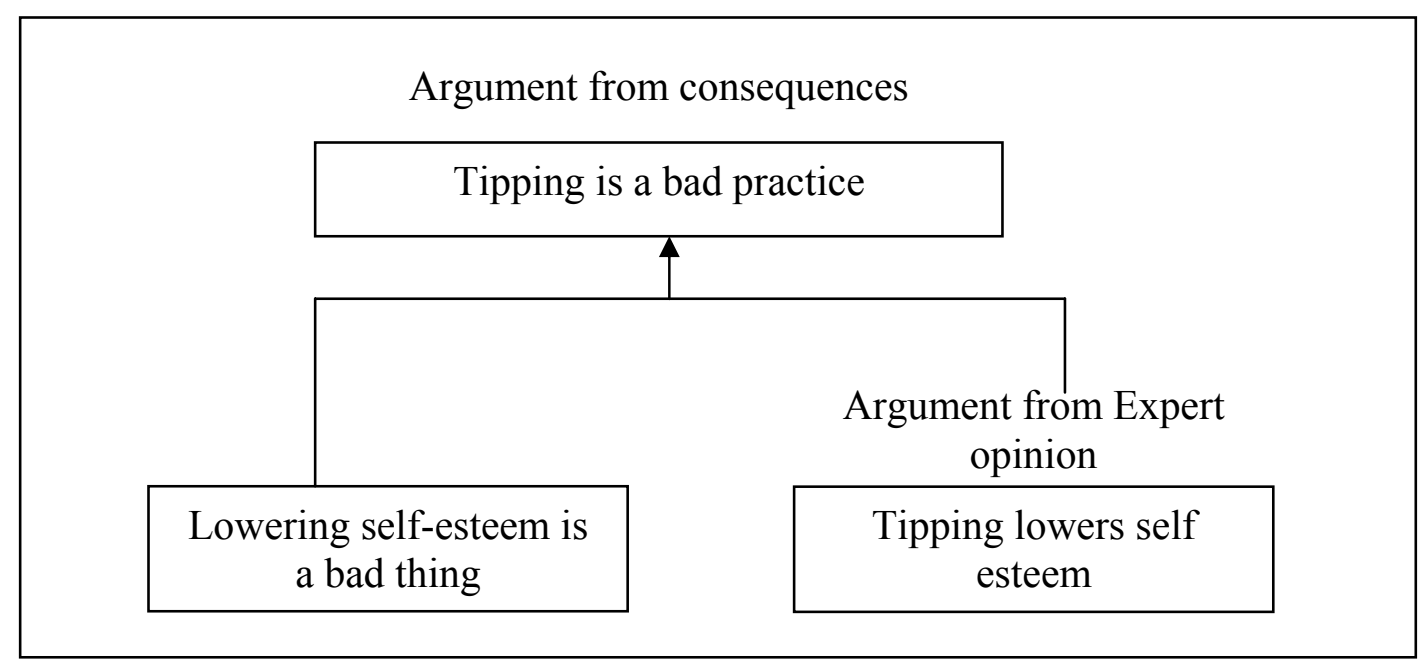

Figure 3. Diagramming the self-esteem argument

Argument schemes are not classified according to their logical form but according to their content. Many argument schemes in fact express epistemological principles (such as the scheme from the expert opinion) or principles of practical reasoning (such as the scheme from consequences). Accordingly, different domains may have different sets of such principles. Each argument scheme comes with a customized set of critical questions that have to be answered when assessing whether their application in a specific case is warranted. Consequently, each different premise is associated with its own particular types of attack, in contrast to the purely logical systems in which attacks are uniform. 
Some of these questions pertain to acceptability of the premises, such as "is the expert $E$ in the position to know about the proposition A?". Other critical questions point at exceptional circumstances in which the scheme may not apply, such as "is $E$ sincere?" or "are there better ways to bring about these good consequences?". Clearly, the possibility to ask such critical questions makes argument schemes defeasible, since negative answers to such critical questions are in fact counterarguments, such as "Expert $E$ is not sincere since he is a relative of the suspect and relatives of suspects tend to protect the suspect".

Reed, Rowe and Walton developed a system, called Araucaria System (Reed and Rowe, 2001), (Reed and Walton, 2003) in order to construct an online repository of arguments drawn from newspaper editorials, parliamentary reports and judicial summaries. Using argumentation schemes, the result of any given analysis is a marked up version of the original text. That is, the text is interspersed with tags that indicate which parts of the text correspond to individual propositions, how these propositions relate to others, where particular argumentation schemes are instantiated, where enthymematic premises should be inserted and how a particular claim is evaluated by the analyst. The format of this markup is described by the Argument Markup Language, AML, described in detail in (Reed and Rowe, 2001). The Araucaria System that creates files marked up according to AML is a tool of informal logic. As such, it can be employed as an aid to support argument analysis and as a diagrammatic presentation tool. An extension of the Argument Interchange Format (Chesnevar et al., 2006) to represent argumentation schemes has been also defined in (Rahwan et al., 2007).

The argumentation schemes have the following advantages and limits:

\section{Advantages}

- They illustrate the structure of the arguments using real cases as examples $(+\mathrm{C} 1)$.

- They can be used for knowledge representation using diagrams (+ C4).

- They can be extended for knowledge elicitation (+ C4).

- They model the inference and the defeasible rules using the critical questions (+ C5). 
- They consider different acceptability criteria that are related to the nature of the schema (+ C7).

\section{Limits}

- They are based on an informal logic that does not define the defeasible rules and the argumentation relations $(-\mathrm{C} 2)$.

- The interaction between the different argumentation schemes is not specified. Although the authors claimed that these schemes can be used to model the argumentation process, the dialectical structure of the schemes is not clearly addressed and formalized ${ }^{1}(-\mathrm{C} 2)$.

- The criterions used in the taxonomy of the argumentation schemes are not specified but only based on the practical observations. In addition, this taxonomy is not exhaustive (- C3).

- They only emphasize the structure of the arguments without taking into account the participants' knowledge bases (- C6).

\subsection{Anscombre and Ducrot's Approach}

Anscombre and Ducrot (1983) emphasized a linguistic phenomenon called "the obligation to conclude" by studying the relationship between statements or propositions. Mainly, they studied the argumentative instructions involved in the use of argumentative connectors such as but and however in natural language, for example: "The weather is nice, but I am tired".

Ducrot (1991) indicates: "The speaker, after having uttered the first proposition $p$, expects the addressee to draw a conclusion $r$. The second proposition $q$, preceded by a $b u t$, tends to avoid this conclusion by signaling a new fact that contradicts it. The whole movement would be: " $p$; you are thinking of concluding $r$; do not do so, because $q$ ". In our example, we can think of a rule saying that 'If the weather is nice, then I will go out', but the connector "but" cancels the expected conclusion "then I will go out". The authors

\footnotetext{
${ }^{1}$ Some recent publications have considered this limit, particularly (Rahwan et al., 2007) and (Gordon et al., 2007).
} 
introduced this notion to represent the argumentative notion of rebuttal. In our example, the but-sentence "but I am tired" is a rebuttal for the conclusion that could have been drawn from the information "The weather is nice". To formalize this obligation to conclude, Anscombre and Ducrot (1983) studied the internal structure of arguments associating a claim to a conclusion. They used the term topos to indicate this structure. The arguments of a topos can be associated with a grade taken on an argumentative scale (Anscombre, 1995) as illustrated by the following topos: The better the weather is, the more you should go out.

The topos-based approach has been used to study argumentative discourse (Moeshler, 1985) and to represent gradual knowledge in several knowledge-based applications such as knowledge acquisition, knowledge validation and explanations (Galarreta and Trousse, 1996) (Raccah, 1996). Dieng (1989) used this approach to generate qualitative explanations about the inferences made by an expert system applied to dyke design. Instead of presenting the quantitative formulas used in the expert system's rules, explanations used topos structure to adapt the corresponding information to the user.

The advantages and the limits of Anscombre and Ducrot's Approach can be summarized as follows:

\section{Advantages}

- The notion of topos can be used to illustrate and formalize the link between the premises and the conclusion of an argument $(+\mathrm{C} 1)$.

- The approach can be used to generate qualitative explanations about the inference rules $(+\mathrm{C} 5)$.

\section{Limits}

- The approach is defined in an informal language and the different argumentation relations (defense, undercut, etc.) are not defined (- C2).

- The theoretical foundations are not clearly stated (- C3).

- It does not offer a deep mechanism for knowledge representation (- C4). 
- It neglects the agents participating in the argumentation game (- C6).

- It cannot be used for knowledge elicitation because it highlights the structure of an argument without distinguishing the different elements and without specifying the acceptability criterion and because it does not illustrate the argumentation process (- C2, - C4, - C7).

\subsection{Breton's Model}

Breton (1996) proposed an argumentation model highlighting the importance of the reasoning type: deduction or analogy. He proposed the concept of double argumentative relaxation, which consists in considering the construction of an argument as a two-stage process: (1) a stage of framing the reality, which provides a frame where the defended opinion can be inserted (ground preparation); (2) a stage of linking this reality and the defended opinion. On the basis of this notion, Breton proposed a classification of arguments (Figure 4). This classification is based on the distinction between framing or realignment arguments and linkage arguments. Framing arguments allows the construction of a reference reality in which the addressee can accept the speaker's proposition. These arguments correspond to the preparation of the argument content. Some of these arguments, called authority arguments, rest on what is already known. They use notions like competences, experiences or testimonies. Other arguments, more innovative, propose a new representation of the reality: they are the realignment arguments of reality. They are based on definitions, presentations, associations (which consist in performing new connections between different notions), or dissociations (which consist in distinguishing the different facets of the same concept). 


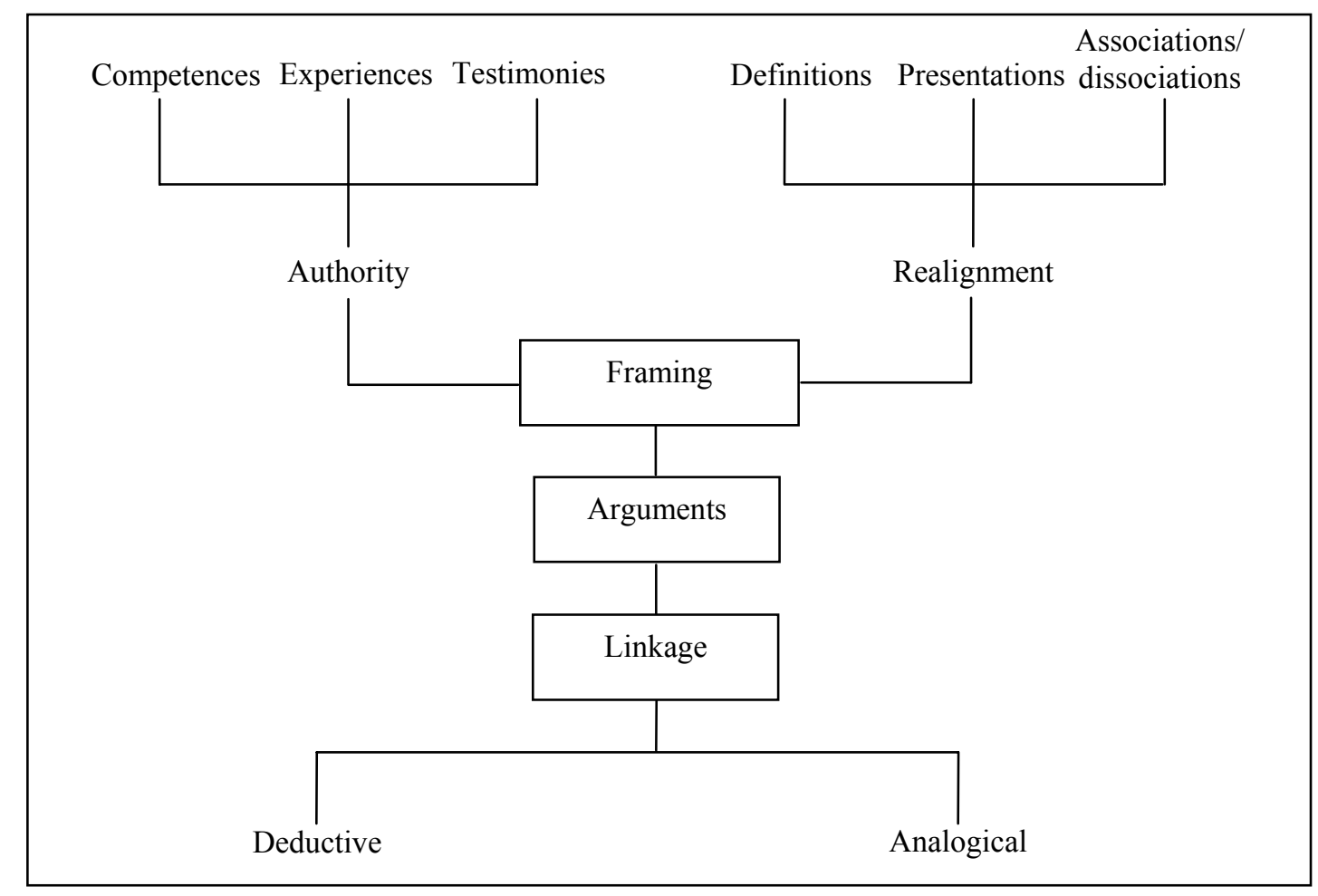

Figure 4. Breton's Model

The purpose of linkage arguments is to insert the defended opinion in the representation of the reality. Breton distinguished two types of these arguments: deductive arguments and analogical arguments. The deductive arguments make it possible to build a continuous logic chain between the reference reality and the defended opinion. This continuity does not characterize the analogical arguments. However, analogical arguments aim at linking the framing reality and the suggested opinion. The metaphor, the comparison, the analogical comparison and the argument by the example are examples of analogical arguments.

Breton's model has the following advantages and limits:

\section{Advantages}

- It provides a general taxonomy of arguments by defining the link between a macro-view and a micro-view of arguments (+ C5). 
- It models the different levels in the acceptability of arguments and inference mechanism (Authority level, Realignment, deductive level and analogical level) $(+\mathrm{C} 5,+\mathrm{C} 7)$.

\section{Limits}

- It does not offer any definition of the argument structure and it does not specify the argumentation process. Consequently, this model is not appropriate for knowledge representation and elicitation (- $\mathrm{C} 1,-\mathrm{C} 2,-\mathrm{C} 4)$.

- The theoretical foundations are missing (- C3).

- It does not take into account the characteristics of the participating agents (- C6).

\subsection{Other Models}

Alvarado and Dyer (1985) postulated the existence of argument units as basic constructs of argument knowledge, which consists of configurations of attack and support relationships related to abstract goal and plan situations. Argument units allow a language understanding system to recognize and interpret arguments in various domains. This work aimed at modeling how a refutation or accusation is organized and how this affects the process of comprehension, memory construction, and question answering. This approach manages several different knowledge sources, including scripts, goals, plans, actions, beliefs. Alvarado implemented a prototype computer program (called OPED), capable of reading editorial segments in the domain of politico-economics, and answering questions about their argument content.

Konolige and Pollack (1989, 1993) used an argumentative background to capture interesting properties of the theory of intention. They approached cognitive attitudes of belief and knowledge by providing a representationalist model of intention. The resulting formalism is useful for tasks such as plan recognition, in which one agent must determine the mental state of another agent using partial information. This model of intention would replace the traditional normal modal logics (in which an agent believes all the consequence of his beliefs). 


\subsection{Comparison}

In this section, we compare the monological models of argumentation presented above. Table 1 illustrates this comparison. It is based on the following elements:

- Argument structure: which are the components of an argument?

- Argumentation process: how the argumentation process is supported?

- Argument types: which argument types does the model support?

- Inference rules: does the model specify the inference rules?

\section{Dialogical Models}

Monological models of argumentation focus on structural relationships between arguments. On the contrary, formal dialectics proposes dialogical structures to model the connectedness of utterances. Dialogical models focus on the issue of fallacious arguments, i.e., invalid arguments that appear to be valid. They are rule-governed structures of organized conversations in which two parties, or more, speak in turn in an orderly way. These rules are the principles that govern the participants' acts, and consequently the use of dialectical moves (Gordon, 1994; Loui and Norman, 1995). To assess the models in this category, we provide hereafter a set of evaluation criteria:

\section{Evaluation criteria for dialogical models}

C1: Definition of a formal model of argumentative dialogues: explanation of how the dialogues are specified along with the allowed moves (the protocol).

C2: Definition of the rules under which the dialogue is consistent.

C3: Specification of the relations between the moves: definition of how a move attacks, justifies, etc., another move.

C4: Modeling the evolution of the dialogue.

C5: Distinguishing between argumentative and non-argumentative dialogues.

C6: Clarity of the underlying argumentation theory including the acceptability of arguments and reasoning issues. 
C7: Applicability of the model: definition of the domains in which the model can be applied along with its purposes, particularly in terms of knowledge representation and knowledge elicitation.

C8: Consideration of the participants in the argumentation process: specification of how participants can use the model and how they are modeled, for example in terms of specifying their knowledge bases and social relationships.

C9: Specification of the protocols combination: definition of how the different protocols/dialogues can be combined to model complex dialogues.

\subsection{Formal Dialectics proposed by Hamblin and MacKenzie}

Hamblin (1970) and MacKenzie (1979, 1981) proposed a mathematical model of dialogues called formal dialectics. They defined some connectors necessary to the formalization of the propositional contents of utterances, and a set of locutions for capturing the speech acts performed by participants when conversing. The dialectical system proposed by MacKenzie, and called system DC, is an extension to the one proposed by Hamblin. MacKenzie's DC provides a set of rules for arguing about the truth of a proposition. Each participant, called player, has the goal of convincing the other participant, and can assert or retract facts, challenging the other player's assertions, ask whether something is true or not, and demand that inconsistencies be resolved. When a player asserts a proposition or an argument for a proposition, this proposition or argument is inserted into a public store called commitments store (CS) and is accessible to both participants. There are rules which define how the commitment stores are updated and whether particular illocutions can be uttered at a particular time. 


\begin{tabular}{|c|c|c|c|c|}
\hline & $\begin{array}{l}\text { Argument } \\
\text { structure }\end{array}$ & $\begin{array}{c}\text { Argumentation } \\
\text { process }\end{array}$ & Argument types & Inference rules \\
\hline $\begin{array}{l}\text { Toulmin and its } \\
\text { extensions }\end{array}$ & $\begin{array}{l}\text { Data, qualifier, } \\
\text { claim, different } \\
\text { types of warrants, } \\
\text { backing and } \\
\text { rebuttal }\end{array}$ & $\begin{array}{l}\text { Partially supported } \\
\text { using Toulmin's } \\
\text { dialogue game }\end{array}$ & $\begin{array}{l}\text { Convergent } \\
\text { arguments, linked } \\
\text { arguments, } \\
\text { arguments in } \\
\text { sequence and } \\
\text { divergent } \\
\text { arguments }\end{array}$ & $\begin{array}{l}\text { Partially } \\
\text { specified using } \\
\text { warrants, backing } \\
\text { and rebuttal }\end{array}$ \\
\hline $\begin{array}{c}\text { Reed and } \\
\text { Walton }\end{array}$ & $\begin{array}{l}\text { Major premise, } \\
\text { minor premise } \\
\text { and conclusion }\end{array}$ & Not specified & $\begin{array}{l}\text { Several argument } \\
\text { types }\end{array}$ & $\begin{array}{l}\text { Specified using } \\
\text { critical questions }\end{array}$ \\
\hline $\begin{array}{l}\text { Anscombre and } \\
\text { Ducrot }\end{array}$ & Topoï & Not specified & Not specified & $\begin{array}{l}\text { Specified using } \\
\text { topos: gradual } \\
\text { inference rules }\end{array}$ \\
\hline Breton & Not specified & Not specified & $\begin{array}{l}\text { Authority } \\
\text { arguments, } \\
\text { realignment } \\
\text { arguments, } \\
\text { deductive } \\
\text { arguments and } \\
\text { analogical } \\
\text { arguments }\end{array}$ & $\begin{array}{l}\text { Specified using } \\
\text { framing and } \\
\text { linkage. }\end{array}$ \\
\hline Other models & $\begin{array}{l}\text { Support and } \\
\text { attack patterns }\end{array}$ & $\begin{array}{l}\text { Supported using } \\
\text { units }\end{array}$ & Not specified & $\begin{array}{l}\text { Specified using } \\
\text { argument entities }\end{array}$ \\
\hline
\end{tabular}

Table 1. Comparison of the monological models

A MacKenzie's dialectical system mainly consists of:

1. A set of moves: they are linguistic acts, for example assertions, questions, etc.

2. A commitment store: defined at the level of each player, this store makes it possible to keep the trace of the various phases of the dialogue. 
3. A set of dialogue rules: they define allowed and prohibited moves. These rules have the following form "if condition, moves $C$ are prohibited". A dialogue is said to be successful when the participants conform to these rules.

The language used in DC contains propositional formulas: “ $p$ ", “ $\neg p$ " and " $p \vee q "$. Locutions are constructed from communicative functions that are applied to these propositions. For example, the moves: "question(fine)" and "assertion (fine, fine $\rightarrow$ hot)" indicate respectively the question "is it fine?" and the assertion "the weather is fine, and when the weather is fine, the weather is hot".

Table 2 illustrates the evolution of the CSs of two players $A$ and $B$ during the following dialogue:

A1: The doctors cannot make this surgery

B2: Why?

A3: Because the patient is too old and that he refuses it

B4: Why does he refuse?

A5: Because there is little chance of success.

\begin{tabular}{|l|l|l|l|l|}
\hline \multicolumn{1}{|c|}{ Turn } & Player & \multicolumn{1}{|c|}{ Move } & \multicolumn{2}{|c|}{$\operatorname{CS}(B)$} \\
\hline 1 & $A$ & Assert $(\neg d)$ & $\neg d$ & $\neg d$ \\
2 & $B$ & $\operatorname{Challenge}(\neg d)$ & $\neg d$ & $? \neg d$ \\
3 & $A$ & $\operatorname{Assert}(p \wedge \neg a)$ & $\neg d, p \wedge \neg a, p \wedge \neg a \rightarrow \neg d$ & $? \neg d, p \wedge \neg a, p \wedge \neg a \rightarrow \neg d$ \\
4 & $B$ & Challenge $(\neg a)$ & $\neg d, p \wedge \neg a, p \wedge \neg a \rightarrow \neg d$ & $? \neg d, p, ? \neg a, p \wedge \neg a \rightarrow \neg d$ \\
5 & $A$ & Assert $(s)$ & $\neg d, p \wedge \neg a, \mathrm{~s}, p \wedge \neg a \rightarrow \neg d$, & $? \neg d, \mathrm{p}, ? \neg \mathrm{a}, \mathrm{s}, \mathrm{p} \wedge \neg \mathrm{a} \rightarrow \neg \mathrm{d}$, \\
& & & $s \rightarrow p$ & $\mathrm{~s} \rightarrow \mathrm{p}$ \\
\hline
\end{tabular}

Table 2. The evolution of CSs during a dialogue

The dialogue starts with $A$ 's assertion $(\neg d)$ : "the doctors cannot make this surgery". Thus, $A$ commits itself and commits its adversary $B$ to this fact. Thereafter, $B$ challenges this assertion (one speaks in this case about disengagement on the fact and an engagement on the challenge). After that, $A$ provides a justification, which commits the 
two players to this assertion and to the fact that this assertion logically implies the challenged fact. The dialogue continues in a similar way with $B$ 's challenge of an $A$ 's justification part, which leads $A$ to propose a new justification.

\section{Advantages}

This model has the following advantages:

- It provides a mathematical model of the dialogue identifying the allowed moves that the players can play at each turn $(+\mathrm{C} 1)$.

- It identifies the cases in which the dialogue is not consistent, for example if a player asserts $p$ at turn $i$ and asserts $q \rightarrow \neg p$ at turn $j(i<j)(+\mathrm{C} 2)$.

- It captures the evolution of the dialogue $(+\mathrm{C} 4)$.

\section{Limits}

The limits of this model are:

- It does not provide any logical relation between the different moves, for example a move which attacks, or justifies another move (- C3).

- It does not distinguish between argumentative and not argumentative dialogues (C5).

- It does not illustrate the reasoning aspects of the players (- C6).

- It only allows a symbolic representation of knowledge used in the dialogue using a propositional language $( \pm \mathrm{C} 7)$.

- It cannot be used for knowledge elicitation because it does not address the structure of arguments and how they can be constructed (- C7).

- It does not consider the participants' models and their social relationships (- C8).

- It does not specify combination of different dialogues (- C9)

\subsection{Amgoud et al.'s Model}

Several researchers have suggested using argumentation techniques in order to model and analyze negotiation dialogues (Sycara, 1990), (Parsons and Jennings, 1996), (Parsons et al., 1998), (Tohmé, 1997) (Rahwan et al., 2004). Amgoud and her colleagues (Amgoud et al., 2000a, 2000b) extended these proposals by investigating the use of argumentation for 
a wider range of dialogue types according to the classification of Walton and Krabbe (1995). The six main types of dialogues proposed by Walton and Krabbe are:

1. Persuasion, which is centered on conflicting points of view.

2. Negotiation, in which participants aim to achieve a settlement that is particularly advantageous for individual parties.

3. Inquiry, in which the aim is to collectively discover more information, as well as to destroy incorrect information.

4. Deliberation, which is driven by the need to take a collective decision.

5. Information-seeking, in which one party asks for information known by another.

6. Eristic, in which two parties combat each other in a quarrel.

This classification is based upon two factors: the initial situation and the goal of the dialogue. Table 3 illustrates these factors.

\begin{tabular}{|l|l|l|}
\multicolumn{1}{|c|}{ Dialogue type } & \multicolumn{1}{c|}{ Initial situation } & \multicolumn{1}{c|}{ Dialogue goal } \\
\hline Persuasion & Conflicting point of view & Resolution of conflict \\
\hline Negotiation & Conflict of interest & Making a deal \\
\hline Inquiry & General ignorance & Growth of knowledge \\
\hline Deliberation & Need for action & Reach a decision \\
\hline Information-seeking & Personal ignorance & Spreading knowledge \\
\hline Eristic & Antagonism & $\begin{array}{l}\text { Accommodation } \\
\text { relationship }\end{array}$ \\
\hline
\end{tabular}

Table 3. Walton and Krabbe’s dialogue types classification

The approach proposed by Amgoud et al. (Amgoud et al., 2000a, 2000b) relies upon MacKenzie's formal dialectics. The dialogue rules of this system are formulated in terms of the arguments that each player can construct. Dialogues are assumed to take place between two agents, $P$ and $C$, where $P$ is arguing in favor of some proposition, and $C$ counter-argues. Each player has a knowledge base ( $\Sigma P$ and $\Sigma C$ respectively), containing his beliefs. As in DC, each player has access to another knowledge base accessible to both players, containing the commitments made during the dialogue. These commitment stores are denoted $C S(P)$ and $C S(C)$ respectively. The union of the commitment stores 
can be viewed as the state of the dialogue at turn $t$. All the knowledge bases described above contain propositional formulas and are not closed under deduction.

Both players are equipped with an argumentation system. Each has access to his own private knowledge base and to both commitment stores. The two argumentation systems are then used to help players to maintain the coherence of their beliefs, and thus to avoid asserting things which are defeated by other knowledge from $C S(P) \cup C S(C)$. In this sense the argumentation systems help to ensure that the players are rational.

To model the dialogue types proposed by Walton and Krabbe, the authors used seven dialogue moves: assert, accept, question, challenge, request, promise and refuse. For each move, they defined rationality rules, dialogue rules, and update rules. The rationality rules specify the preconditions for playing the move. The update rules specify how commitment stores are modified by the move. The dialogue rules specify the moves that the other player can make next, and so specify the protocol under which the dialogue takes place. Figure 5 presents these rules for the assert and challenge moves.

$\operatorname{assert}(\boldsymbol{p})$ where $p$ is a propositional formula.

Rationality the player uses its argumentation system to check if there is an acceptable argument for the fact $p$.

Dialogue the other player can respond with:

$$
\begin{aligned}
& \text { 1: } \operatorname{accept}(p) \\
& \text { 2: } \operatorname{assert}(\neg p) \\
& \text { 3: challenge }(p)
\end{aligned}
$$

Update $C S_{i}(P)=C S_{i-l}(P) \cup\{p\}$ and $C S_{i}(C)=C S_{i-l}(C)$

challenge(p) where $p$ is a propositional formula.

Rationality $\varnothing$

Dialogue the other player can only assert (S) where $\mathrm{S}$ is an argument supporting $p$.

Update $C S_{i}(P)=C S_{i-l}(P)$ and $C S_{i}(C)=C S_{i-1}(C)$

Figure 5. Example of rationality, dialogue and update rules

The authors showed that this framework can be used to implement the language for persuasive negotiation interactions proposed by Sierra et al. (1998). In (Parsons et al., 2002), this approach is used to analyze formal agent dialogues using the dialogue 
typology proposed by Walton and Krabbe. The authors defined a set of locutions by which agents can trade arguments and a set of protocols by which dialogues can be carried out. In (Parsons et al., 2003), this approach is used to examine the outcomes of the dialogues that an argumentation system permits, for example what agents come to accept during the course of the dialogue.

To capture the idea that some facts are more strongly believed (or desired, or intended, depending on the nature of the facts) the authors assume that any set of facts has a preference order over it. This preference derives from the stratification of the knowledge base $\Sigma$ into non-overlapping sets $\Sigma 1, \ldots, \Sigma$ such that facts in $\Sigma i$ are all equally preferred and are more preferred than those in $\Sigma j$ where $j>i$. The preference level of a nonempty subset $H$ of $\Sigma$, denoted level $(H)$, is the number of the highest numbered layer which has a member in $H$.

Definition. Let $(H 1, h 1)$ and $(H 2, h 2)$ be two arguments. $(H 1, h 1)$ is preferred to $(H 2$, h2) iff level $(H 1) \leq \operatorname{level}(H 2)$.

Using this notion of preference, an argumentation system can be defined as follows:

Definition. An argumentation system is a triple $<A(\Sigma)$, Attack, Prefs such that $A(\Sigma)$ is a set of the arguments built from $\Sigma$, Attack is a binary relation representing defeat relationship between arguments, Attack $\subseteq A(\Sigma) \times A(\Sigma)$, and Pref is a (partial or complete) pre-ordering on $\mathrm{A}(\Sigma) \times \mathrm{A}(\Sigma)$. $>^{\text {Pref }}$ stands for the strict pre-order associated with Pref.

Using preference relations between arguments, Amgoud and Cayrol (2000) accounted for two complementary views on the notion of argument acceptability: acceptability based on the existence of direct counter-arguments and acceptability based on the existence of defenders. An argument is said to be acceptable if it is preferred to its direct defeaters or if it is defended against its defeaters. Using this framework, Amgoud and Hameurlain (2007) proposed an approach to select moves using a two steps decision process. The purpose of the first step is to select among all the act types allowed by the selected 
protocol, the best option which satisfies the most important strategic goals of the agent. During the second step, the participant agent selects among different alternatives, the best content that will accompany the act and that satisfies the agent's functional goals.

\section{Advantages}

This model has the following advantages:

- It models the different types of dialogues $(+\mathrm{C} 1)$.

- It captures the evolution of the dialogue along with its consistency $(+\mathrm{C} 2,+\mathrm{C} 4)$.

- It is based on an argumentative theory that explains the reasoning aspects of the players (+ C6).

- It models the relations between arguments (+ C6).

- It provides a technique for knowledge representation using dialogue trees $(+\mathrm{C} 7)$.

\section{Limits}

This model has three main limits:

- Although it captures the relations between arguments, it does not capture the argumentative relations between the moves (- C3).

- It does not distinguish between argumentative and non-argumentative dialogues (C5).

- It cannot be directly used for knowledge elicitation because it only considers the attack relation between arguments without addressing the other possible relations and the structure of arguments $( \pm \mathrm{C} 7)$.

- It does not distinguish between agents' internal arguments and the external arguments used by the agents in their conversations (- C8).

- It does not consider the social relations between the players (- C8).

- It does not explain how the different protocols can be connected to build complex protocols. For example, the model does not specify how a persuasion dialogue can be connected to a negotiation or a deliberation dialogue (- C9). 


\subsection{Bentahar et al.’s Model}

Bentahar, Moulin and Chaib-draa (2004a) extended the approach proposed by Amgoud and her colleagues by proposing an approach combining public (observable) aspects and argumentative components of dialogues. In this approach, agents participate in dialogues by performing actions on social commitments. A social commitment is a public commitment made by an agent (the debtor), that some fact is true or that something will be done. This commitment is directed toward a set of agents (creditors). A commitment is an obligation in the sense that the debtor must respect and behave in accordance to this commitment. Commitments are social in the sense that they are expressed publicly. Consequently, they are different from the private mental states like beliefs, desires and intentions (BDI) that are used in pure mentalistic approaches such as the BDI approach. In order to model the dynamics of conversations, a speech act is defined as an action performed on a commitment or on its content. A speech act is an abstract act that an agent, the speaker, performs when producing an utterance and addressing it to another agent, the addressee. The actions that an agent can perform on commitments are: Act $\in\{$ Create, Withdraw $\}$. The actions that an agent can perform on commitment contents are: Act-content $\in\{$ Accept, Refuse, Challenge, Defend, Attack, Justify\}.

According to this approach, before committing to some fact $h$ being true (i.e. before creating a commitment whose content is $h$ ), the speaker agent must use its argumentation system to build an argument supporting $h$. On the other side, the addressee agent must use its own argumentation system to select the answer it will give (i.e. to decide about the appropriate manipulation of the content of an existing commitment). For example, an agent $A g_{1}$ accepts the commitment content $h$ proposed by another agent if $A g_{1}$ has an argument for $h$. If $A g_{l}$ has an argument neither for $h$, nor for $\neg h$, then it challenges $h$. The authors also distinguished between arguments that an agent possesses (private arguments) and arguments that this agent used in the conversation (public arguments).

On the basis of this approach, the authors proposed a framework called Commitment and Argument Network. This framework allows the representation of knowledge used by agents when conversing. This knowledge is represented by actions that agents perform 
when conversing. The argumentation phases in a dialogue between software agents (justification, attack and defense) are represented as actions applied to social commitment contents. The formalism also offers means that enable agents to take part in consistent conversations. Agents jointly build the network that represents their conversation as it progresses. This allows the agents to make sure at any time that the conversation is consistent and to determine which speech act to perform on the basis of the current state of the conversation. Consistency is ensured by the relationships existing between different commitments, different argumentation relations and different actions (creation, acceptance, fulfillment, etc.). The formal semantics of this framework is defined using a hybrid logic combining Computation Tree Logic CTL* and dynamic logic (Bentahar et al., 2004c; Bentahar et al. 2007c; Bentahar et al., 2009b). In (Bentahar et al., 2007b; Bentahar et al., 2009a), the authors extended this approach by proposing a mechanism allowing agents to reason about their strategies and tactics. The authors argued that strategic reasoning enables agents to decide about the global communication plan in terms the macro-actions to be performed in order to achieve the main conversational goal, and that tactical reasoning allows agents to locally select, at each moment, the most appropriate argument according to the adopted strategy.

An implementation of a persuasion dialogue game protocol between two agents $A g_{l}$ and $\mathrm{Ag}_{2}$ based on this framework is described in (Bentahar et al., 2004b). In this system the persuasion dynamics is described by the chaining of a finite set of dialogue games: acceptance move, refusal move, defense, challenge, attack, and justification games. Several games can be played in parallel (Figure 6).

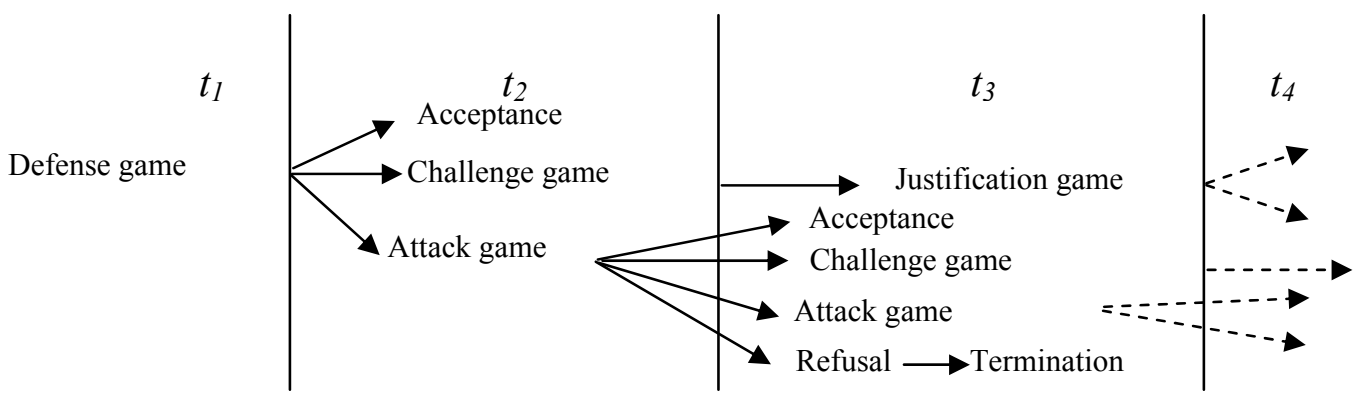

Figure 6. The persuasion dialogue dynamics 
After $A g_{1}$ 's defense game at moment $t_{1}, A g_{2}$ can, at moment $t_{2}$, accept a part of the arguments presented by $A g_{l}$, challenge another part, and/or attack a third part. These games are played in parallel. At moment $t_{3}, A g_{l}$ answers the challenge game by playing a justification game and answers the attack game by playing an acceptance move, a challenge game, another attack game, and/or a final refusal move. The persuasion dynamics continues until the exit conditions become satisfied (final acceptance or a refusal). An extension of this protocol has been applied to web services technology (Bentahar et al., 2007a), and to persuasive negotiation (Bentahar and Labban, 2009). This protocol plays the role of the dialectical proof theory of the argumentation system.

\section{Advantages}

This model has the following advantages:

- It provides a model for dialogues considering consistency and dynamics issues (+ $\mathrm{C} 1,+\mathrm{C} 2)$.

- It captures the evolution of the dialogue not only in terms of the moves that are played during the dialogue, but also in terms of the argumentative and non argumentative relations between the communicative acts $(+\mathrm{C} 3,+\mathrm{C} 4,+\mathrm{C} 5)$.

- It captures the agents' trustworthiness as an acceptability criterion of arguments ( \pm C6).

- It captures the reasoning aspects of the participating agents (+ C6).

- It allows a representation of knowledge using commitment and argument network $(+\mathrm{C} 7)$.

- It distinguishes between the internal agents' arguments and the observed arguments used in the dialogue $(+\mathrm{C} 8)$.

\section{Limits}

The limits of this model are:

- It does not consider the preferences that can exist between arguments ( $\pm \mathrm{C} 6)$.

- It cannot be directly used for knowledge elicitation because it only offers an external representation of argumentative dialogue without tacking into account the micro-structure of arguments $( \pm \mathrm{C} 7)$. 
- It does not illustrate the combination of the different dialogue types (- C9).

\subsection{Dung's Model}

According to Dung (1995), the idea of argumentation-based reasoning is that a dialectical statement is believable if it can be argued successfully against attacking arguments. Thus, the beliefs of a rational agent are characterized by the relations between its "internal arguments" supporting its beliefs and the "external arguments" supporting contrary beliefs. In this context, argumentation is the process of constructing arguments for propositions representing an agent's beliefs. While in standard logic an argument represents a definite proof, in defeasible reasoning an argument may represent just a provisional reason to believe a proposition: it does not provide a proof, but only a plausible support to the proposition. Building on the fact that different arguments may support contradictory conclusions, the main problem is to determine which conclusions should be believed in the presence of conflicting arguments.

Dung proposed a framework for the semantics of extended logic programming with explicit negation. An extended logic program $P$ is composed of clauses of the form:

$L 0 \leftarrow L 1, \ldots, L \mathrm{~m}$, not- $L m+1, \ldots$, not- $L m+n$, where $L i$ are literals or negation of literals. And not-Li are the assumptions on which the proof is based. Since the acceptance of a proof depends on the acceptance of the assumptions on which it is based, arguing for a conclusion means arguing for the assumptions on which some proof of the conclusion is based. An argument $A$ supports a literal $L$ if there exists a proof of $L$ based on assumptions contained in $A$. An argument is self-defeating if it supports both $L$ and $\neg L$. Dung distinguishes two ways of attacking an argument:

- An argument $A$ attacks an argument $A$ ' via reductio ad absurdum (RAA-attack) if $A \cup A^{\prime}$ is self-defeating.

- An argument $A$ attacks an argument $A$ ' via ground attack (g-attack) if there is an assumption not- $L$ in $A$ ' such that $L$ is supported by $A$.

\section{Example}

Consider the following extended logic program: 
innocent $(X) \leftarrow \operatorname{not} \operatorname{guilty}(X)$

guilty $(X) \leftarrow$ confess guilt $(X)$

$\neg$ innocent $(X) \leftarrow$ confess guilt $(X)$, not lies $(X)$

confess guilt( john); lies (john); confess guilt( peter)

Consider the sets of assumptions $A 0=\varnothing, A 1=$ not-guilty(john) $\}, A 2=$ \{notlies(peter) $\}$, and $A 3=$ \{not-guilty(peter) $\}$. Then the argument $A 0$ supports guilty(john), the argument $A 1$ supports innocent(john), the argument $A 2$ supports $\neg$ innocent( peter). Note that the argument $A 2$ for $\neg$ innocent(peter) RAA-attacks the argument $A 3$ for innocent( peter), since $A 2 \cup A 3$ is self-defeating, supporting both innocent(peter) and $\neg$ innocent(peter). The argument $A 0$ for guilty(john) is a ground attack for the argument A1 for innocent(john).

\section{Advantages}

The two main advantages of this model are:

- It models the argumentative structure of the dialogue and its consistency $(+\mathrm{C} 1,+$ $\mathrm{C} 2$ ).

- It provides a general approach for logical programming based on the argumentation. Therefore, the framework can be implemented as a logical program (+ C6).

- It offers a logical theory of acceptability of arguments based on the relations between arguments $(+\mathrm{C} 6)$.

\section{Limits}

The model has the following limits:

- It does not specify the relations between the dialogue moves (- C3)

- It does not capture the evolution of the dialogue, but only its argumentative structure without distinguishing argumentative moves from non-argumentative ones (- C4, - C5).

- It only allows a symbolic representation of knowledge using propositions and the attack relation $( \pm \mathrm{C} 7)$. 
- It cannot be directly used for knowledge elicitation because it only considers the attack relation between arguments without addressing the other possible relations and the structure of arguments $( \pm \mathrm{C} 7)$.

- It does not take into account the characteristics of the participating agents in the argumentative games (- C8).

- The acceptability of arguments is only based on the logical relations between arguments without taking into account the social relations between agents or the preferences between arguments (- C8).

- It does not illustrate how the different dialogues can be modeled (- C9).

\subsection{Simari and Loui's Model}

Simari and Loui (Simari, 1989), (Simari and Loui, 1992) presented a system in order to characterize the conditions under which an argument structure is preferred to another. An Agent's knowledge is represented by a pair $(K, \Delta)$. The set $K$ represents indefeasible knowledge, which should be consistent. $\Delta$ is a finite set of defeasible rules. Defeasible rules are expressed in a meta-language. A defeasible rule of the form $p \longmapsto q$ is used to represent that " $p$ is a reason for $q . "$

Simari and Loui define a meta-relationship that relates formulas of $K$ and ground instances of $\Delta$ with a formula $h$. This meta-relationship is called defeasible consequence, and it represents a derivation from $K$ to $h$ using ground instances of $\Delta$, which can be regarded as material implications for the application of modus ponens. The symbol $\vdash$ stands for the classical inference and $\perp$ stands for false. A subset $T$ of ground instances of $\Delta$ 's members is an argument for a formula $h$ if and only if:

1. $K \cup T \vdash h(T$ derives $h)$

2. $K \cup T \nvdash \perp(T$ is consistent with regard to $K)$

3. $\forall T^{\prime} \subset T: K \cup T^{\prime} \nvdash h$ ( $T$ is minimal.)

The first condition indicates that the formula $h$ can be derived from the indefeasible knowledge $K$ using the defeasible rules described in $T$. The second condition indicates the consistence of $T$ because it is not possible to derive impossible things from $K$ using the 
rules described in $T$. The third condition indicates that $\mathrm{T}$ is minimal because $h$ cannot be derived from any subset of $T$ (but only from $T$ ).

An argument $T$ and a formula $h$ in the conditions defined above comprise an argument structure, written $\langle T, h>$. Arguments contain sub-arguments. The argument structure $<S$, $j>$ is a sub-argument of the argument structure $<T, h>$ if $S \subseteq T$.

\section{Example}

Suppose that we are provided with the following knowledge about cars:

- Cars that are reliable are usually recommendable.

- Cars that are reliable but no longer produced are usually not recommendable.

- European cars for which there are spare parts are usually reliable.

- Cars with cooling problems are usually not reliable.

- Cars with a rear motor usually have cooling problems.

- Cars with a rear motor usually have rear-wheel drive.

- Cars that have low consumption and rear-wheel drive are usually reliable.

- Italian cars are European cars.

Suppose that we know that $f 6$ is an Italian car which has spare parts available, has a rear motor and low consumption, and that it is no longer produced.

This knowledge can be modeled in Simari and Loui's framework by a set:

$\mathrm{K}=\{$ rear motor $(f 6)$, italian $(f 6)$, low cons $(f 6)$, no longer produced $(f 6)$, has spare $\operatorname{parts}(f 6), \operatorname{italian}(X) \rightarrow$ european $(X)\}$

and a set $\Delta$ of defeasible rules, defined as follows:

$\Delta=\{\operatorname{reliable}(X) \longmapsto$ recommendable $(X)$,

reliable $(X) \wedge$ no-longer produced $(\mathrm{X}) \longmapsto \neg$ recommendable $(X)$,

$\operatorname{european}(X) \wedge$ has-spare-parts $(X) \longmapsto \operatorname{reliable}(X)$,

$\operatorname{rear-motor}(X) \longmapsto \operatorname{rear}-\operatorname{system}(\mathrm{X})$,

$\operatorname{low}$-cons $(\mathrm{X}) \wedge$ rear-system $(\mathrm{X}) \longmapsto \operatorname{reliable}(X)$, 
rear-motor $(X) \longmapsto$ cooling-problems $(X)$,

cooling $\operatorname{problems}(X) \longmapsto \neg$ reliable $(X)\}$

Some of the different arguments that can be built from this knowledge are $A 1$ for recommendable( $f 6), A 2$ for $\neg$ reliable $(f 6)$ where:

$A 1=\{$ european $(f \quad 6) \wedge$ has-spare-parts $(f \quad 6) \longmapsto$ reliable $(f \quad 6)$, reliable $(f \quad 6) \longmapsto$ recommendable $(f 6)\}$

$A 2=\{$ rear-motor $(f 6) \longmapsto$ cooling-problems $(f 6)$,

Cooling-problems $(f 6) \longmapsto \neg$ reliable $(f 6)\}$

An argument can be depicted by a treelike structure, as shown in Figure 7.

Arguments can be in conflict in different ways. The binary relationships of disagreement, Counter-argumentation, and defeat are defined as follows:

- Two arguments $<A 1, h 1>$ and $<A 2, h 2>$ disagree if and only if $K \cup\{h 1, h 2\} \vdash \perp$.

- Given two arguments $\langle A 1, h 1>$ and $\langle A 2, h 2>,\langle A 1, h 1>$ counter-argues $\langle A 2, h 2>$ if and only if there exists a sub-argument $\langle A, h>$ of $\langle A 2, h 2>$ such that $\angle A 1, h 1>$ and $<A$, $h\rangle$ disagree. The sub-argument $\langle A, h\rangle$ of $\langle A 2, h 2\rangle$ is called the disagreeing subargument.

- Given two arguments $<A 1, h 1>$ and $<A 2, h 2>,<A 1, h 1>$ defeats $<A 2, h 2>$ if and only if there exists a sub-argument $<A, h>$ of $<A 2, h 2>$ such that: $<A 1, h 1>$ counter-argues $<A$, $h>$. if $\langle A 1, h 1>$ defeats $\langle A 2, h 2>$, we also say that $<A 1, h 1>$ is a defeater for $<A 2, h 2>$.

To model the justification process, Simari and Loui proposed the notion of dialectical tree. Formally, a dialectical tree for $\langle A, h\rangle$, denoted $T\langle A, h\rangle$, is recursively defined as follows: (1) A single node containing an argument $<\mathrm{A}, h>$ with no defeaters is by itself a dialectical tree for $\langle\mathrm{A}, h>$. (2) Suppose that $<\mathrm{A}, h>$ is an argument with defeaters $<A 1$, $h 1>,<A 2, h 2>, \ldots,<A \mathrm{n}, h n>$. We construct the dialectical tree $T<\mathrm{A}, h>$ by putting $<A, h>$ as the root node of it and by making this node the parent node of the roots of the dialectical trees of $\langle A 1, h 1>,\langle A 2, h 2>, \ldots,\langle A \mathrm{n}, h \mathrm{n}>$. Any path, $\lambda=[\langle A 0, h 0\rangle,\langle A 1$, $h 1>, \ldots,<A \mathrm{k}, h \mathrm{k}>]$ in a dialectical tree $T<A, h>$ is called argumentation line. 


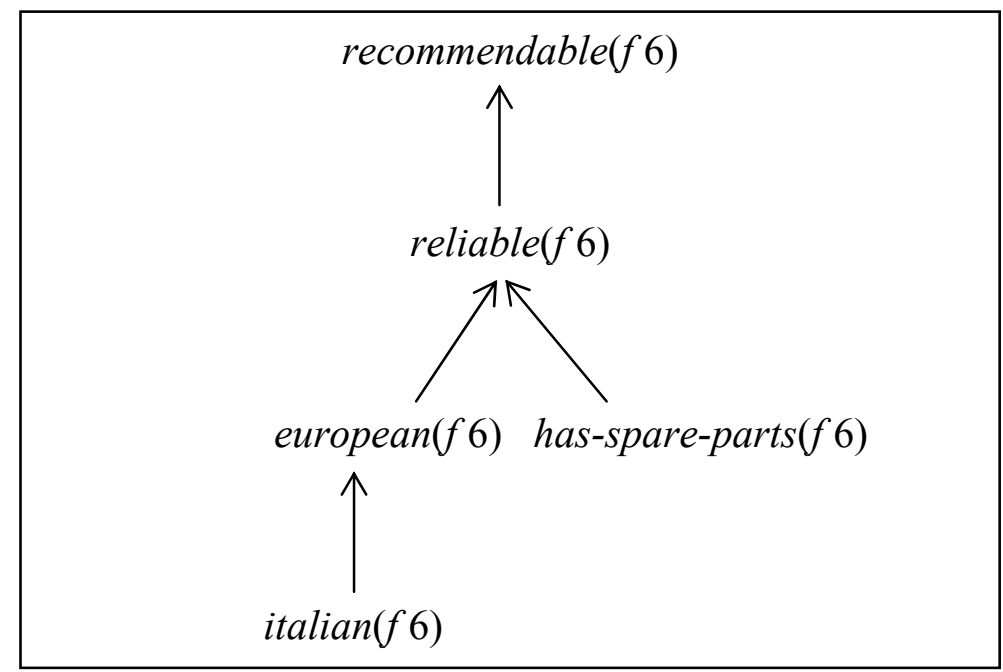

Figure 7. Treelike structure of argument $<A 1$, recommendable $(f 6)>$ in Simari and Loui's framework

The framework proposed by Simari and Loui and refined by Simari and Garcì (1995) was used in various works: Falappa (1999) proposed a belief revision system using a special kind of argument; Bodanza and Simari (1995) analyzed defeasible reasoning in disjunctive databases; Delrieux (1995) introduced a system of plausible reasoning; Augusto (1998) studied the construction of arguments for temporal reasoning. Kraus and his colleagues (1995) proposed a logic of argumentation to reason under uncertainty. In this framework, propositions are labeled with a representation of arguments supporting their validity. The authors define strength mappings from sets of arguments to a selected set of linguistic qualifiers. Hence, propositions can be assigned to classes such as certain, confirmed, probable, plausible, supported and open. This approach provides a uniform framework incorporating a number of numerical and symbolic techniques to assign subjective confidences to propositions on the basis of their supporting arguments. Building on Simari and Loui's framework, Garcìa and Simari (2004) developed a defeasible logic programming system (DeLP) which combines logic programming with defeasible reasoning. The formalism is somewhat similar to the one developed by Prakken and Sartor (1997) in that it is based on the application of rules. 


\section{Advantages}

The main advantages of this model are:

- It provides a structure (dialectical tree) that illustrates the argumentative moves in a dialogue and the relations between these moves, which can be used to decide about the dialogue consistency $(+\mathrm{C} 1,+\mathrm{C} 2,+\mathrm{C} 3)$.

- It captures the dialogue evolution (+ C4).

- It offers a dialectical argumentation theory illustrating the relations between arguments and a logical acceptability criterion $(+\mathrm{C} 6)$.

- It allows a representation of knowledge using dialectical tree $(+\mathrm{C} 7)$.

- It takes into account the beliefs of the agents participating in a dialogue $(+\mathrm{C} 8)$.

\section{Limits}

This model has the following limits:

- The dialectical tree emphasizes only the argumentative structure of the moves, but it does not present the other non-argumentative moves (- C5).

- It cannot be used for knowledge elicitation because it only considers the dialogical structure of arguments $( \pm \mathrm{C} 7)$.

- The acceptability of arguments is only based on the logical relation between arguments without taking into account the social relations between agents or the preferences between arguments $( \pm \mathrm{C} 8)$.

- It does not illustrate how the different dialogues can be modeled (- C9).

\subsection{Rescher's Model}

In his book Dialectics: A Controversy-Oriented Approach to the Theory of Knowledge, the philosopher Rescher (1977) described formal disputation as a process that involves three parties: a proponent, asserting a certain proposition, an opponent able to challenge the proponent's position, and the determiner, which decides whether the proponent's position was successfully defended or not. The disputation proceeds in terms of rules and assertions. The structure of a disputation takes the form of matched responses in which the root is the proponent's initial thesis. Each move in the disputation process consists of one or more fundamental moves. Three kinds of fundamental moves are distinguished: 
- Categorical assertions, which are used to establish that a proposition is true.

- Cautious assertions, which are used to maintain that a proposition is compatible with what have been shown.

- Provisoed assertions, which are used to establish conditional assertions in that if a proposition is true, then usually another proposition is true also.

The first move of a disputation is always a categorical assertion. The possible countermoves to a categorical assertion are a challenge, a cautious denial, or a provisoed denial. The responses to a cautious assertion can be a categorical counter-assertion, or a provisoed counter-assertion. The only way to attack provisoed assertions is to present another provisoed assertion that is equally or better informed than the first one. Two possible attacks are identified by the author: weak distinction, which means that attacking the proposition using a cautious assertion, or strong distinction, which means that attacking the proposition using a categorical assertion.

Simari, Chesnevar, and Garcìa (1994) considered the role of dialectics in defeasible argumentation. They considered some of Rescher's ideas within the Simari and Loui's framework (Simari and Loui, 1992). The resulting evolved framework, called MTDR, is able to cope with fallacious argumentation. Using MTDR as a basis, the authors developed a knowledge representation formalism for defeasible logic programming and implemented a logic programming language to deal with defeasible rules. Chesnever and Simari (Chesnevar, 1996), (Chesnevar and Simari, 1998) analyzed strategies for speeding up the inference process by pruning the search space for arguments to determine whether an argument supports a justified belief.

\section{Advantages}

The dialectical game proposed by Rescher has the two following advantages:

- It is based on a philosophical theory of the disputation, which studies the different rules that each participant must respect $( \pm \mathrm{C} 1, \pm \mathrm{C} 6, \pm \mathrm{C} 8)$. 
- It emphasizes the defeasible rules existing between assertions. Although these rules are defined in an informal logic, they can be used to develop a defeasible logic programming (Chesnevar, 1996) $(+\mathrm{C} 2,+\mathrm{C} 3)$.

\section{Limits}

This model has the following limits:

- It does not specify how the determiner can decide about the termination of a dialectical game (- $\mathrm{C} 4)$.

- It does not offer any structure illustrating the evolution of the dialogue (- C4).

- It only specifies the disputation-based dialogues, but it does not consider the other argumentative dialogues (- C5).

- It does not specify how an argument can be accepted (- C6).

- It only allows a symbolic representation of knowledge using some logical connectors $( \pm \mathrm{C} 7)$.

- It cannot be used for knowledge elicitation ( $\pm \mathrm{C} 7$ ).

- It does not consider the participants' knowledge bases (- C8).

- It does not consider dialogues combination (- C9).

\subsection{Lin and Shoham's Model}

Lin and Shoham (1989) proposed an abstract argument system and showed how wellknown systems of default reasoning could be reformulated in their framework. The scheme is entirely based on inference rules, which are defined over an unspecified logical language. An inference rule can be a base fact of the form $A$, where $A$ is a well-formed formula; it can be a monotonic rule of the form $A 1, \ldots, A n \rightarrow B$, with $n>0$ and where the $A i$ and $B$ are well-formed formula; or it can be a non-monotonic rule of the form $A 1, \ldots$, $A n \Rightarrow \mathrm{B}$, with $n>0$ and where the $A i$ and $B$ are well-formed formula. Basic facts represent explicit knowledge, monotonic rules represent deductive knowledge, and nonmonotonic rules represent common sense knowledge. The three kinds of inference rules are used to build arguments. An argument is a rooted tree with labeled arcs. Arguments must be constructed in such a way that the same formula does not appear 
more than once. An argument supports a formula if the formula is the root of the tree that comprises the argument.

The system is very general, and many issues remain unspecified: for example there is no notion of preference between conflicting arguments. Lin and Shoham introduced an important concept, namely the concept of argument structure. A set $T$ of arguments is an argument structure if the following conditions are satisfied:

1. If $p$ is a base fact, then $p \in T$.

2. $T$ is closed, i.e., $\forall p \in T$, if $p^{\prime}$ is a sub-tree of $p$ then $p^{\prime} \in T$.

3. $T$ is monotonically closed, i.e., if $p$ is built up from $p 1, \ldots, p$ n $\in T$ by a monotonic rule, then $p \in \mathrm{T}$.

4. $T$ is consistent, i.e., it does not contain arguments that support both $\Phi$ and $\neg \Phi$ for any well-formed formula $\Phi$.

The above conditions for the definition of an argument structure can be strengthened by the completeness condition. An argument system is complete with respect to $\Phi$ if either it contains an argument that supports $\Phi$ or it contains an argument that supports $\neg \Phi$.

The authors showed how to use the resulting framework to capture default reasoning, autoepistemic logic, and negation as failure. They also showed how their framework can be applied to formalize inheritance systems, temporal projection and temporal diagnosis. This apparent power may be due to the simplicity of the system, since they do not consider many notions which are fundamental for a working argumentation system as for example preference criteria between conflicting arguments.

\section{Advantages}

This model has the following advantages:

- It uses a simple formal model $( \pm \mathrm{C} 1)$.

- It captures the default reasoning using only some inference rules ( \pm C6). 


\section{Limits}

The limits of this model are:

- It does not illustrate how dialogues can be modeled using the argumentation structure (- C1, -C2, -C4, -C5, -C9).

- It does not define the notions of attack and defense between arguments (- C3).

- It does not specify how agents can participate in dialogues using their reasoning capabilities (- C8).

- It does not specify the acceptability of arguments (- C6).

- Because it is an abstract argumentation system, this model cannot be used for knowledge representation and elicitation (- C7).

\subsection{Other Models}

Flowers et al. (1982) and Birnbaum (1982) investigated the representation of argument structures. They considered arguments as networks of propositions connected by support or attack relations. They analyzed argument structures by identifying commonly occurring patterns of support and attack that encompassed several propositions. They used argument entities as basic entities to specify which propositions were worth trying to attack or support. Thus, argument entities could be used to create plan rebuttals, and to generate expectations about an opponent's possible rebuttals. They concluded that arguments possess useful structural properties, abstracted from the specific propositions they encompass. This model has the advantage of illustrating the dynamics of the argumentative process. However, the fundamental notions of the argumentation theory (defense, attack, undercut, etc.) are not specified. In addition, the agents' beliefs and reasoning on the network are not considered.

Vreeswijk $(1993,1995,1997)$ proposed a thoughtful critique of previous argumentation models and presented a new approach to argumentation called abstract argumentation system. An abstract argumentation system is a collection of arguments considered as defeasible proofs that are partially ordered by a relation expressing differences in conclusive force. Difference in conclusive force causes defeat among arguments. The aim of the theory is to find out which arguments eventually emerge undefeated. Vreeswijk's 
theory assumed that the premises of the argumentation process are undefeasible. Baroni and colleagues (2000) extend Vreeswijk's theory by recognizing that premises can be defeated and by relaxing the implicit assumption about their strength. Vreeswijk also formalized the notion of defeasible debate in which there is always a winner and a loser, to determine whether a specific proposition is defendable, assuming an abstract argumentation system in the background. There are two parties a proponent $P$ and an opponent $O$, who may perform three kinds of moves: they may state one or more theses, pose a question, or give an answer. The party stating the theses is the defender of them; the other party is the attacker. A debate turns out to be a (possibly infinite) sequence of moves such that theses are followed by questions which are followed by answers, which themselves are possibly followed by theses.

The model proposed by Vreeswijk has the advantage of modeling the evolution of the argumentative dialogues using the notion of defeasible debate and the relation expressing the differences in conclusive force. However, this model is too abstract to be used for eliciting and representing knowledge and for modeling the different types of dialogues. The reason is that the components of the model are specified in an unstructured language and the relations between arguments are not clearly defined. In addition, the acceptability of the arguments does not take into consideration the trustworthiness or the social position of the players.

Verheij (1996) analyzed the properties and roles of rules and reasons in argumentation. He studied the conditions in which an argument should be defeated at a particular stage of the argumentation process. He developed CumulA, a formal model of defeasible argumentation in stages in which arguments are seen as treelike structures of sentences describing the way a conclusion is supported. The purpose of CumulA is to study how the status of an argument is determined by its structure, the associated counterarguments, and the stage reached in an argumentation process.

Schroeder (1999) followed the work done by Dung (1995) and Prakken and Sartor (1997) on logic programming and formal argumentation systems. Shroeder used two types of 
negation: implicit negation not $a$ to express the lack of evidence for $a$, and the explicit negation $\neg a$ to state that there is an explicit proof for $\neg a$. An extended logic program is a (potentially infinite) set of rules in the form $L 0 \leftarrow L 1, \ldots, L l$, not $L l+1$, not $L m(0 \leq l \leq$ $m$ ) where each $L i$ is a literal. Literals of the form not $L l$ are called default literals. Let $P$ be an extended logic program. An argument for a conclusion $L$ is a finite sequence $\mathrm{A}=$ $\left[r_{s}, r_{t}\right]$ of ground instances of rules $r_{i} \in P$ such that several conditions hold (Schroeder, 1999). Schroeder defined the notions of undercut and rebut, attack and defeat of arguments in the same way. Building on this, Schroeder (2000) developed Ultimate Ratio: a visualization tool of argumentation. The objective of the Ultimate Ratio Project (Schroeder, 2000) was to visualize the argumentation process in a form understandable by people. To this end, the author used the dynamic construction of proof trees as a metaphor for the process of argumentation. The tree construction is a representation of the argument and of the control flow in the logic program. The system provides an interactive animation of the argumentation process of a single agent, allowing the user to navigate through the argumentation space as well as the argumentation process which is displayed as a 3D tree. In addition, the user can navigate through the static space of all possible arguments.

Bench-Capon and his team used the concept of argument to investigate the defeasibility of explanation in legal expert systems and the construction of arguments through dialog games (Bench-Capon et al. 1993). Rissland and her team (1993) developed several systems which facilitate argument creation, using a case-based approach. Loui and Norman (1995) defined different criteria to formalize rationales within an argumentative framework. A rationale for a rule is a structure which contains relevant additional information about the reasons for rule adoption, whereas the rationale for a case is additional information about the decision reached. Sillince (1994) investigated conflict resolution between software agents which attempted to make claims using tactical rules (such as fairness and commitment). Agents might support inconsistent beliefs until another agent is able to attack their beliefs with a strong argument. 
In his theory of defeasible reasoning, Pollock (1991) postulated that reasoning operates in terms of reasons. Reasons can be assembled to comprise arguments. He distinguishes two kinds of reasons, non-defeasible and defeasible. The notion of defeasible reason, also known as prima facie reason, is defined in terms of a special kind of knowledge called defeaters. Defeaters are new reasons that attack the justificatory power that a certain (defeasible) reason has on behalf of its conclusion. Pollock refers to two kinds of defeaters: rebutting defeaters and undercutting defeaters. A rebutting defeater is a reason that attacks a conclusion by supporting the opposite proposition, while an undercutting defeater is a reason that attacks the connection existing between a reason and a conclusion. Building on this, Pollock $(1992,1995)$ proposed a formalism supporting rebutting as well as undercutting defeaters using a preferred-semantics style of overall justification. On the basis of this formalism, a defeasible reasoner: OSCAR System has been implemented. The inference engine of OSCAR can be seen as an implementation of Pollock's theory of defeasible reasoning. OSCAR's reasoning engine is designed to support not only defeasible logic, but also non-defeasible logic. When being used for non-defeasible logic, it basically acts as a theorem-prover for first-order logic. In the OSCAR defeasible component, the status of the arguments (justified or defeated) can change back or forward with every inference step.

Prakken and Sartor (1996) proposed a defeasible argumentation framework supporting different logical notions of legal arguments. The underlying language contained strong literals and weak literals. Strong literals are atomic first-order formulas which can be preceded by classical negation. Weak literals take the form $\sim L$, where $L$ is a strong literal. Informally, $\sim L$ reads as "there is no evidence that $L$ is the case," while $\neg L$ says " $L$ is definitely not the case." Weak negation is used to represent assumptions. Rules have the form: $r: L 0 \wedge \ldots \wedge L j \wedge \sim L k \wedge \ldots \wedge \sim L m \Rightarrow L n$ where $r$ is the name of the rule, and each $L i$ is a strong literal. An argument is defined as a finite sequence $A=[r 0, \ldots, r n]$ of ground instances of rules, such that for every $i(0 \leq i \leq n)$, for every strong literal $L j$ in the antecedent of $r i$, there is a $k<i$ such that $L j$ is the consequent of $r k$, and no two distinct rules in the sequence have the same consequent. A literal $L$ is a conclusion of $A$ if and only if $L$ is the consequent of some rule in A. Like in Pollock's theory, Prakken and 
Sartor distinguished two cases of attack rebutting and undercutting. An argument rebuts another argument if they produce complementary conclusions and a preference for the first argument over the second one can be induced from the priorities defined on the rules that comprise the arguments. An argument undercuts another argument if the first argument has a conclusion which is the strong negation of an assumption of the second argument. The notion of defeat is defined as follows. Given two arguments $A 1$ and $A 2$, $A 1$ defeats $A 2$ if and only if 1) $A 1$ undercuts $A 2$, or 2) $A 1$ rebuts $A 2$ and $A 2$ does not undercut $A 1$. A fundamental notion in Prakken and Sartor's theory is the dialectical proof theory. A proof of a formula takes the form of a dialogue tree, where each branch of the tree is a dialogue and the root of the tree is an argument for the formula. The idea is that every move in a dialogue consists of an argument, where each stated argument attacks the last move of the opponent in a way that meets the player's burden of proof. The required force of each move is motivated by the definition of acceptability based upon the provability justification of the argument used to support the move. An argument $A$ is said to be provably justified if and only if there is a dialogue tree with $A$ as its root, won by the proponent. A player wins a dialogue tree if and only if it wins all branches of the tree. Prakken and Sartor also showed that all provably justified arguments are justified, and that if an argument is provably justified, then all its sub-arguments are provably justified.

The models presented in this section (Verheij, Schroeder, Bench-Capon, Pollock and Prakken and Sartor) have the following advantages and limits:

\section{Advantages}

- They are based on logic programming and/or formal argumentation. Consequently, they take into account the participants' rationality $( \pm \mathrm{C} 1,+\mathrm{C} 6)$.

- They model the relations between arguments/moves using a preferred semantics $(+\mathrm{C} 3)$.

- They illustrate the process of argumentation using structural and procedural arguments or proof trees, which captures dialogue evolution $(+\mathrm{C} 4,+\mathrm{C} 6)$.

- Some of these models can be used for knowledge representation using structural arguments and proof trees $( \pm \mathrm{C} 7)$. 


\section{Limits}

- They do not specify how different dialogue types, for instance deliberation and negotiation, can be modeled (- C1).

- Dialogues consistency is not considered (- C 2).

- They do not distinguish argumentative from non argumentative dialogues (- C5).

- The acceptability of arguments is only based on the defeasible rules and the preference between arguments without considering the social positions of the participants $( \pm \mathrm{C} 6)$.

- They cannot be used for knowledge elicitation because they do not consider the micro-structure of arguments and how these arguments can be produced (- C7).

- They do not indicate how the participants' knowledge bases are presented and updated (- C8).

- They do not consider dialogues combination (- C9).

\subsection{Comparison}

In this section, we compare the dialogical models of argumentation discussed above. Table 4 illustrates this comparison. It is based on the following elements:

- Argumentation notions: which are the argumentation relations the model supports?

- Acceptability criteria: which are the acceptability criteria of arguments the model specifies?

- Argumentation process dynamics: which is the means the model uses in order to illustrate the dynamics of the argumentation process?

- Argumentative dialogues: which are the argumentative dialogue types that can be modeled?

Agent reasoning: does the model take into account the reasoning of the participating agents? 


\begin{tabular}{|c|c|c|c|c|c|}
\hline & $\begin{array}{l}\text { Argumentation } \\
\text { notions }\end{array}$ & $\begin{array}{c}\text { Acceptability } \\
\text { criteria }\end{array}$ & $\begin{array}{c}\text { Argumentation } \\
\text { process } \\
\text { dynamics }\end{array}$ & $\begin{array}{l}\text { Argumentative } \\
\text { dialogue types }\end{array}$ & $\begin{array}{c}\text { Mechanisms } \\
\text { for agent } \\
\text { reasoning }\end{array}$ \\
\hline $\begin{array}{c}\text { Hamblin } \\
\text { and } \\
\text { MacKenzie }\end{array}$ & Not defined & Not defined & $\begin{array}{l}\text { Supported using } \\
\text { the commitment } \\
\text { stores }\end{array}$ & $\begin{array}{l}\text { Persuasion, but } \\
\text { other types can } \\
\text { also be } \\
\text { modeled }\end{array}$ & $\begin{array}{l}\text { Not } \\
\text { considered }\end{array}$ \\
\hline $\begin{array}{l}\text { Amgoud et } \\
\text { al. }\end{array}$ & $\begin{array}{l}\text { Defense, attack, } \\
\text { undercut }\end{array}$ & $\begin{array}{l}\text { Logical } \\
\text { criteria and } \\
\text { preference } \\
\text { criteria }\end{array}$ & $\begin{array}{l}\text { Supported using } \\
\text { the commitment } \\
\text { stores }\end{array}$ & $\begin{array}{l}\text { Walton and } \\
\text { Krabbe } \\
\text { dialogues }\end{array}$ & $\begin{array}{l}\text { Considered } \\
\text { (beliefs and } \\
\text { knowledge } \\
\text { bases) }\end{array}$ \\
\hline $\begin{array}{c}\text { Bentahar et } \\
\text { al. }\end{array}$ & $\begin{array}{l}\text { Defense, attack, } \\
\text { justification }\end{array}$ & $\begin{array}{l}\text { Logical } \\
\text { criteria and } \\
\text { trust criteria }\end{array}$ & $\begin{array}{l}\text { Supported using } \\
\text { the commitment } \\
\text { and argument } \\
\text { network }\end{array}$ & $\begin{array}{l}\text { Persuasion, but } \\
\text { other types can } \\
\text { also be } \\
\text { modeled }\end{array}$ & $\begin{array}{l}\text { Considered } \\
\text { (beliefs and } \\
\text { knowledge } \\
\text { bases) }\end{array}$ \\
\hline Dung & $\begin{array}{l}\text { Defense, attack, } \\
\text { undercut }\end{array}$ & $\begin{array}{l}\text { Logical } \\
\text { criteria }\end{array}$ & Not supported & Not considered & $\begin{array}{l}\text { Not } \\
\text { considered }\end{array}$ \\
\hline $\begin{array}{l}\text { Simari and } \\
\text { Loui }\end{array}$ & $\begin{array}{l}\text { Disagreement, } \\
\text { Counter- } \\
\text { argumentation, } \\
\text { defeat }\end{array}$ & $\begin{array}{l}\text { Logical } \\
\text { criteria }\end{array}$ & $\begin{array}{l}\text { Supported using } \\
\text { the Dialectical } \\
\text { tree }\end{array}$ & Not considered & $\begin{array}{l}\text { Considered } \\
\text { (beliefs) }\end{array}$ \\
\hline Rescher & $\begin{array}{l}\text { Categorical, } \\
\text { cautious and } \\
\text { provisoed } \\
\text { assertions }\end{array}$ & Not specified & Not supported & $\begin{array}{l}\text { disputation- } \\
\text { based dialogues }\end{array}$ & $\begin{array}{l}\text { Not } \\
\text { considered }\end{array}$ \\
\hline $\begin{array}{l}\text { Lin and } \\
\text { Shoham's }\end{array}$ & $\begin{array}{l}\text { nonmonotonic } \\
\text { rules, support }\end{array}$ & Not specified & Rooted tree & Not considered & $\begin{array}{l}\text { Not } \\
\text { considered }\end{array}$ \\
\hline $\begin{array}{c}\text { Other } \\
\text { Models }\end{array}$ & $\begin{array}{l}\text { Defense, attack, } \\
\text { undercut, rebut }\end{array}$ & $\begin{array}{l}\text { Logical } \\
\text { criteria }\end{array}$ & $\begin{array}{l}\text { Structural and } \\
\text { procedural } \\
\text { arguments, } \\
\text { proof trees }\end{array}$ & $\begin{array}{l}\text { Defeasible } \\
\text { debates }\end{array}$ & $\begin{array}{l}\text { Not } \\
\text { considered }\end{array}$ \\
\hline
\end{tabular}

Table 4. The comparison of the dialogical model 


\section{Rhetorical Models}

The research on argumentation presented in the previous section has concentrated on formal dialectics. Another field of argumentation in artificial intelligence focuses on discourses, which are rhetorically argumentative. This field, called rhetorical argumentation, deals with arguments, which are both based on the audience's perception of the world, and with evaluative judgments rather than with establishing the truth of a proposition (Grasso, 2002; Pasquier et al., 2006; Gordon et al., 2007). The following dialogue from (Grasso, 2002) illustrates this idea:

A: Do you like cooking?

B: Not especially. [...] Cooking feels to me like a lot of effort for something (i.e. eating) that's over quite quickly. Also, I often feel tired at the end of a day's work and don't want to spend too much time in the kitchen.

A: You do not cook just because you have to eat! Cooking can be a very relaxing and interesting activity, better than watching TV!

B: I know you're right but that still doesn't make it easy to do!

According to Lodder (1999), logic alone does not suffice to model argumentation. A model should also consider the rhetoric nature of argumentation. He proposed DiaLaw: a formal model in which the logical and rhetorical aspects of legal argumentation are combined. Logic is used to determine whether premises are sufficient to justify the conclusion. DiaLaw stresses a new type of reasoning called diarational reasoning that captures the fact that some conclusions are accepted by the audience without the premises being sufficient. In his model, Lodder (1997) showed the importance of both structural and procedural arguments. A structural argument indicates an explicit relation between two statements. A procedural argument is a statement that, itself or in combination with other non-structural arguments, contributes to the acceptance of another statement, without using a structural argument. For example, procedural arguments are often used in courts. To justify a decision, the authority invokes a series of statements and then links the conclusion to these statements without making explicit the exact relations between the statements and the conclusion. Partly, this approach is comparable to Perelman and 
Olbrechts-Tyteca (1969). The main difference is that DiaLaw does not reject the use of logic.

\section{Evaluation criteria for rhetorical models}

We use the following criteria to assess the most significant rhetorical models proposed in the literature:

C1: Representation of arguments: specification of how rhetorical arguments are represented.

C2: Specification of relations between arguments or rhetorical concepts.

C3: Clarity and relevance of the theoretical foundations: consideration of the foundations used in the definition of arguments and their structures.

C4: Applicability of the model: definition of the domains in which the model can be applied along with its purposes.

C5: Wiliness of the model for knowledge elicitation.

\subsection{The Models proposed by Olbrechts-Tyteca and by Grasso}

In Aristotle's rhetorical argumentation (Ryan, 1992), the emphasis is put on the audience rather than on the argument itself. In a persuasive dialogue, the rhetorician appeals to the audience's set of beliefs in order to try to persuade this audience, rather than to achieve general acceptability. Using Aristotle's definition, philosophers Perelman and OlbrechtsTyteca (1969) proposed a new rhetoric theory aiming at identifying discursive techniques. Based on an approach that goes from examples to generalization, this theory proposes a collection of argument schemas which are successful in practice. This collection is classified in terms of the objects of the argumentation and the types of audience's beliefs that the schema exploits. Each schema is described by associations of concepts, either known or new to the audience in order to win the audience's acceptance. A rhetorical schema is meant to express when it is admissible to use a given relationship between concepts (this concept will be detailed in the following subsection). This notion of argumentation schemas is different from the schemes proposed by Reed and Walton (Section 3.3) in the sense that the later do not consider the audience's beliefs. They are also different in terms of the elements used to classify the identified schemes (the 
elements used by Olbrechts-Tyteca are presented in the following subsection). However, the two notions have the same purpose which is to capture common and stereotypical patterns of reasoning.

Grasso used this theory to propose a framework for rhetorical argumentation (Grasso, 2002) and a mental model for a rhetorical arguer (Grasso, 2003). The purpose is to build artificial agents able to engage in rhetorical argumentation. In this framework, argumentation aims at reaching an evaluation of an object or of a state of affairs. This evaluation is a way to pass value from one topic to another, in the same way as a deductive argument passes truth from one proposition to another. Formally, we say that there exists an evaluation of a concept $c$, in the set of concepts $C$ from a certain perspective $p$ of a set $P$ from which the evaluation is made, if there exists a mapping $E$ of the pair $(c, p)$ into a set $V$ of values. Assuming that $V$ is a set consisting of two elements: good and bad, we write: $E: C \times P \mapsto V=\{$ good, bad $\}$

In order to model the rhetorical argumentation, Grasso assumed that this argumentation can take place on a certain set of objects of discourse, forming an ontology. The set of relationships between these concepts is denoted $R$. In this context, a relationship is thought of as any of the possible ways in which two concepts may be linked in the ontology. They not only might have a semantic association between them, but one may be the generalization of the other, or they may both contribute to define a third object. The fact that there exists a relationship $r$ between the objects $c$ and $c^{\prime}$ in an ontology is expressed as follows: $r\left(c, c^{\prime}\right)$ where $r \in R$ and $c, c^{\prime} \in C$.

Grasso defined a rhetorical argument as the act of putting forward the evaluation of a concept, on the basis of a relationship existing between this concept and another concept, and by means of a rhetorical schema. If we call $S$ the set of available rhetorical schemas, and $E^{\prime}=C \times P \times V$ the set of all the evaluations, then a rhetorical argument $A_{R}$ can be defined as a function: $A_{R}: E^{\prime}=E^{\prime} \times R \times S \mapsto E^{\prime}$ 
In other words, If we have a concept $c \in C$ and an evaluation of such a concept $E(c, p)$ from a given perspective $p \in P$, we can put forward a rhetorical argument in favor or against a second concept $c^{\prime} \in C$ iff 1) a relationship exists in the ontology between the two concepts $c$ and $c^{\prime} r\left(c, c^{\prime}\right), r \in R$, and 2) a schema can be identified that exploits such a relation $s \in S$.

\section{Rhetorical Schemata}

A rhetorical schema is meant to express when it is admissible to use a given relationship, by capturing the restrictions that, from case to case, have to apply for the relationship to be used legally, or safely. It is important to note that a definition of a rhetorical schema should explicitly represent its admissibility, together with the relationship that the schema is meant to formalize.

Olbrechts-Tyteca (1969) classified common argumentative schemas into two main categories: argumentation by association and argumentation by dissociation. The first category includes: 1) quasi-logical arguments; 2) arguments based on the structure of reality; and 3) arguments founding the structure of reality. Quasi-logical arguments are arguments that look like logical and mathematical proofs but which lack the exact and formal character. Arguments based on a contradiction or incompatibility relation and transitive arguments are examples of quasi-logical arguments. Arguments based on the structure of reality often use the causality relation, while arguments founding the structure of reality use rhetoric relations such as example giving, illustration or analogy. The second category consists of arguments where two concepts that the audience considered as a whole are separated ("you talk of law, I talk of justice").

According to the Acceptability criterion, as rhetorical arguments have to be convincing to the audience, a rhetorical argumentation framework should consider what that audience perceives as true. Hence, the arguments have to be explicitly based on the audience's set of beliefs. In addition, the argument proposed must be Relevant to the discussion. Finally, the argument proposed must be Sufficient for the audience to be able to reach an evaluation, by proposing a not biased and balanced point of view (Groarke et al., 1997). 
Formally, a rhetorical schema is defined as follows:

Definition A rhetorical argumentation schema is a 6-tuple:

$R S=<N, C, O c, A c, R c, S c>$

where: $N$ is the name of the schema, $C$ is the claim the schema supports, $O c$ are the ontological constraints the schema is based on, $A c$ are the acceptability constraints, $R c$ are the relevance constraints, and $S c$ are the sufficiency constraints.

Grasso explicitly differentiated constraint types not only for the sake of clarity, but also for operational purposes. She assumed an ordering of the constraints reflecting their importance in the context of the argument. Four types of constraints have been distinguished:

Ontological Constraints The ontological constraints represent the relationships between the concepts depending on the underlying language. These constraints spell out how the concepts that are used in the rhetorical argument should relate to one another in the ontology.

Acceptability Constraints The acceptability constraints are defined according to the beliefs and perceptions of the audience. Disregarding the audience's beliefs can lead to perfectly valid and sound arguments that might nevertheless be not persuasive enough.

Relevance Constraints To define these constraints, the author used an operational interpretation, by capturing the effort that the listeners have to put in processing the information. She therefore defined the relevance constraints as the representation of which premises have been communicated for the audience to act towards the acceptance (or rejection) of the conclusion. Such premises, or beliefs, should be not only possessed by the audience, but also "accessible" without great effort. This property has been often referred to as being in the "focus of attention" (Grosz and Sidner, 1986), which means that the beliefs should be part of the entities that are salient to what is being said.

Sufficiency Constraints Sufficiency constraints are defined in the informal logic literature as the representation of all the premises that are needed in order for the audience to establish that the conclusion is more likely than not (Groarke et al., 1997). They are meant to measure the speaker's "fairness" in putting forward the argument. In 
non deductive argumentation, it is not always possible or feasible to give precise rules to establish whether this constraint is satisfied. The author adopted a circumscriptive approach by encapsulating in the notion of sufficiency the list that Perelman and Olbrechts-Tyteca proposed for counter-arguments to each schema.

The models proposed by Olbrechts-Tyteca and by Grasso have the following advantages and limits.

\section{Advantages}

- The models represent arguments using schemas (+ C1).

- They have strong theoretical foundation (+ C3).

- They can be used for knowledge representation using the argumentation schemas and the relations between concepts $(+\mathrm{C} 4)$.

- They can be used to implement rational agents able to use a flexible way for argumentation (+ C4).

\section{Limits}

- They do not specify in a systematic way the different relations that can exist between the different concepts (- C2).

- They cannot be used for knowledge elicitation because they do not take into account the structure of arguments and because they do not specify the construction process of these arguments (- C5).

\subsection{A Rhetorical Approach for Persuasive Negotiation}

Ramchurn et al. (2007) addressed the role of rhetorical argumentation in the persuasive negotiation. They presented a rhetorical model of persuasion that defines the main types of rhetorical particles that provide a decision making model. The purpose of such a model is to enable an agent to determine which type of rhetorical argument to send in a given context and how to evaluate rhetorical arguments that are received. 
The proposals of persuasive negotiation proposed by the authors are supported by rhetorical arguments (such as threats, rewards or appeals). By 'rhetorical' the authors mean that the proponent believes that the enactment (in the case of rewards and threats) or validity (in the case of appeals) of the content of these arguments will, in some way, influence the opponent's evaluation of the issues at stake so that the deal being proposed is more likely to be accepted.

The authors defined the persuasive illocutions in a rhetorical sense as they imply that the sender anticipates what the hearer believes rather than looking at the logical defeasibility or truth of the statements. The primary precondition for any illocutionary act to be sent is that the proponent believes that the achievement of the proposal is preferred to its current state. The post-condition is that the recipient $A g 2$ believes that the sender $A g 1$ prefers the proposal to be executed rather than staying in its current state. For example, for an agent $A g 1$ to threaten another agent $A g 2, A g 1$ must believe that $A g 2$ prefers staying in the current state rather than enacting the proposal and that $A g 2$ can be threatened. Also, $A g 1$ must believe that the state brought about by the threat is less preferred by $A g 2$ than the state brought about by the proposal (in the third condition) otherwise, we would not need threats.

Before an agent can choose which argument to send in a given context, it needs to have a way of differentiating between the various rhetorical arguments at its disposal. To deal with this aspect, the authors proposed the notion of rhetorical strength of an argument. A strong argument is one that quickly persuades an opponent to do the proposal, while a weak argument is one that is likely to be less persuasive. Naturally, these notions are viewed from the perspective of the proponent since the actual impact on the opponent will not be known. The authors assumed that the various rhetorical argument types can be ordered in a rigid domain-independent hierarchy of strength (for example threats are more powerful than rewards). There are many factors that can help determine an argument's rhetorical strength, however, two of the most obvious ones are (Tindale, 1999): 
1. Its success rate: the number of times it has caused a proposal to be accepted (i.e. its rhetorical adherence effect).

2. Its effect on the trust that the opponent has in the proponent (it may increase, decrease or remain the same). Increasing trust increases the opponent's tendency to accept subsequent proposals from the proponent.

The key factors that determine which arguments to send are the desirability of the proposal to the proponent and the degree of trust that exists between the two agents. The authors combined these notions using a series of heuristics based on the believed motivations of the recipient to determine which persuasive strength of argument should be chosen. Since these heuristics involve significant degrees of uncertainty, Ramchurn et al. (2007) exploited fuzzy reasoning techniques. Thus, rules of the following form are used:

RULE 1: if trust is low and utility of the proposal is high, then send a strong argument RULE 2: if trust is high and utility of the proposal is low, then send a weak argument The rationale behind these rules is closely related to the concept of argument strength. The stronger an argument is, the more it is likely to lessen the opponent's trust in the proponent, and the more it could coerce the opponent to change his preferences (for example by making a significant threat). However, this lowering of trust results in less cooperative behavior, which in turn, makes it harder for the proponent to persuade the opponent to accept its future proposals. Thus, strong arguments should only be sent, for example, when the negotiation needs to take place in the shortest time possible, when the proposal has a high utility to the proponent or when it is known that the other partner cannot be trusted to reach effective agreements efficiently. Otherwise, weaker arguments should be used.

\section{Advantages}

- The model considers the relations between arguments using rhetorical strength (+ $\mathrm{C} 2$ ).

- It has philosophical and theoretical foundations $(+\mathrm{C} 3)$.

- This approach proposes a concrete example of using rhetorical argumentation in multi-agent systems $(+\mathrm{C} 4)$. 
- The approach can be easily extended for other agent communication types $(+\mathrm{C} 4)$.

\section{Limits}

- It does not consider the representation of arguments (- C1).

- It cannot be used for knowledge elicitation because it does not specify the structure of arguments and the possible relationships between them (- C5).

\subsection{Cabrol-Hatimi's Model}

Cabrol-Hatimi (1999) proposed an argumentation model called MARINE (Modèle d'ARgumentation à INtervalles d'Evaluation) based on the evaluation of the persuasive strength of arguments for the audience. MARINE is a conceptual model that can be used to represent, in a satisfactory way, the structure of a natural argumentation and an associated semantics allowing one to reason on this structure. This formalism aims at modeling the reasoning on the argumentation by taking into account the possible approximations and uncertainties in value judgments.

The model uses a multi-valued approach of the belief concept. What is interesting is the strength of arguments and not only if these arguments are convincing for the audience or not. However, it can be difficult for the user to determine in a precise way the adequate nuance. This problem is solved in MARINE by a representation of the evaluations in the form of intervals included in the interval [0,1], which makes it possible to manage the inaccuracy and uncertainty in the evaluation of all the data. A multi-graduated semantic scale going for example from not convincing, for 0 , to very convincing, for 1 , is proposed to the user in order to facilitate its estimates.

MARINE is based on a distinction between the arguments and the used criteria. A criterion can be considered as the name of an argumentation schema in Reed and Walton's approach (Reed and Walton, 2003). MARINE also distinguishes the concepts of argument and counter-argument and formalizes the arguments with multiple subarguments. In order to illustrate these concepts, let us consider the following example:

p1: I do not have any professional experience in this domain. 
p2: On a purely individual basis, I have experience in the networking field.

p3: I have the required practical competences.

$p 1$ is an argument against $p 3$, based on the professional experience criterion. $p 2$ is an argument for $p 3$ using the individual experience criterion. $p 3$ is the conclusion of an argument with multiple under-arguments. The importance, or the relevance, of an argument is related to the importance of the underlying criterion. The professional experience criterion can for example be more important than the individual experience criterion.

The MARINE model is defined using a logical language whose syntax allows the authors to describe the structure of the argumentation based on well-formed formulas. The semantics of this language enables the authors to express the persuasive strength of a conclusion according to the persuasive strengths and the relevance of its underarguments.

The APLA system (Cabrol-Hatimi and Tazi, 2000) was developed around the argumentation model of MARINA. It includes a module supporting argument choices. This system proposes a satisfactory strategy and evaluates strategies that are selected by the user. It handles a concept of strategy restricted to the choice of a subset of arguments related to the initial argumentation. The purpose is to determine the arguments that should be drawn in relation to the problems of the counter-arguments. Several research works in psychology, psycholinguistics, rhetoric and communication theories, showed that an argumentation is generally more effective if it presents, in addition to the defended thesis, some of the opposite theses. The module supporting the choice of the arguments is based upon the assumption according to which an unfavorable argument for the defended position must be presented if it uses an important criterion, even if one cannot refute it directly. A relevance threshold is thus defined, as well as the concepts of obligatory and optional under-arguments.

The advantages and the limits of Cabrol-Hatimi's model are: 


\section{Advantages}

- The model provides a structure of natural arguments and an associated semantics $( \pm \mathrm{C} 1)$

- It is based on some theoretical approaches $(+\mathrm{C} 3)$.

- It models the agents' beliefs and reasoning on the argumentation structures.

- It takes into account the knowledge representation (+ C4).

\section{Limits}

- The criteria that can be used to characterize an argument are not clearly defined ($\mathrm{C} 1)$.

- It does not specify in a formal way how an argument can support or attack another argument (- C2).

- It is not appropriate for knowledge elicitation because it does not consider the possible relations between arguments (- C5).

\subsection{Other Models}

Inspired by social psychology, Pasquier and his colleagues (2006) developed a new cognitive coherence-based approach to rhetorical argumentation. In this approach, the cognitive issues associated to the production, evaluation and integration of arguments are driven by calculus on a formal framework of the cognitive coherence theory. Such a theory allows modeling the cognitive aspects of communication through the concept of attitude change. Cognitions are represented through two sets of elements: accepted and refused ones. They can be related by positive constraints, which represent positive binary relations such as facilitation, entailment and explanatory relations, or negative constraints, which are negative binary relations such as mutual exclusion and incompatibility relations. A positive constraint is satisfied if the two related elements are both accepted or rejected. A negative constraint is satisfied only if one element is accepted. Two elements are then coherent if they are related by a satisfied constraint and incoherent if they are connected by an unsatisfied constraint. These relations capture the notions of dissonance and consonance in the cognitive coherence theory. In this framework, the authors define argumentation as the processes by which an agent shows 
to the other agents why a given position is coherent. The idea is that agents can construct, exchange and weigh up arguments relevant to conflicting issues when there is a manifest incoherence. The framework takes into account the audience by attempting to reduce cognitive dissonance between the agents.

Based on Walton's argument structure (1996), Gordon et al. (2007) presented the Carneades Argumentation Framework, a mathematical model of argument evaluation applying proof standards to determine the defensibility of arguments and the acceptability of statements on an issue-by-issue basis. The framework uses three types of premises: ordinary premises, presumptions and exceptions, and information about four types of dialectical status of statements: undisputed, at issue, accepted, and rejected in order to model critical questions. These critical questions are used to analyze presumptive argumentation schemes. Those that are attached to an argumentation scheme enumerate possibilities of challenging arguments built using the scheme. Arguments are identified, analyzed and evaluated by fitting premise-conclusion structures that can be identified using argumentation schemes and by a dialectical process. Whether or not a premise of an argument holds depends on whether it is undisputed, at issue, or decided. The ultimate purpose of the framework is to allow the burden of proof to be allocated to the proponent or the respondent.

Dialogical models and rhetorical models deal with arguments used in dialogues (for example, persuasion dialogues). The difference between these two categories is that the first one aims at formalizing the logical relationship between arguments (undercutting defeat, rebutting defeat, justification, etc.) in order to find an acceptable argument, while the second one tries to identify the discursive techniques that can be used in a dialogue. Rhetorical models are frameworks used to model the argumentation process according to the perception of the participants and not on the basis of the argument structures. 


\section{A Case Study: Argumentation Models to Represent the Rationale of Critiquing Courses of Action}

In this section we present a case study which illustrates how the comparison framework proposed in the previous sections has been used to select an argumentation model to represent the knowledge contained in texts critiquing courses of action. Several courses of action (COA) had been proposed by different planning teams during a military exercise in a fictitious situation, and two domain experts had been enrolled in order to critic these courses of action. In order to generate structured critics and to systematically assess certain aspects of the courses of action, a template had been provided to the domain experts. The texts contained in the templates' slots filled in by the experts, constitute the corpus which is the initial material of our case study. Figure 8 provides an extract of such a corpus. The critic of COA partially illustrated in this figure is about a military scenario during a supposed hostility between an alliance (Alliance) and an enemy (two countries are considered as the enemy: Redland (RL) and Whiteland (WL)). The objective is to discuss the advantages and disadvantages of allowing the alliance to seize initiative.

\begin{tabular}{|c|c|c|}
\hline Item in the grid: & \multicolumn{2}{|c|}{ A11: Allows Alliance to seize initiative } \\
\hline COA Name & Advantages & Disadvantages \\
\hline Titanium Overcast & $\begin{array}{l}\text { A11: Allows Alliance to seize } \\
\text { initiative } \\
\text { A12: Higher RL/WL attrition } \\
\text { A13: Faster } \\
\ldots\end{array}$ & $\begin{array}{l}\text { D11: Less flexible } \\
\text { D12: More Alliance Air } \\
\text { casualties } \\
\text { D13: } \\
\ldots\end{array}$ \\
\hline $\begin{array}{l}\text { Detailed description: } \\
\text { A11. } 1\end{array}$ & \multicolumn{2}{|c|}{$\begin{array}{l}\text { The Titanium Overcast COA is one designed to take the offensive } \\
\text { as soon as possible after the commencement of hostilities. Phase } \\
2 \text {, which takes place following D-Day aggressively, moves air } \\
\text { forces into RL and WL airspace in order to gain air superiority } \\
\text { and force RL and WL air forces to be reactive. By seizing the } \\
\text { initiative in this way, Alliance air forces should force RL/WL air } \\
\text { forces into a defensive posture and thus limit their ability to } \\
\text { support RL Army operations. }\end{array}$} \\
\hline $\begin{array}{l}\text { Explanation of why this is } \\
\text { considered as an advantage } \\
\text { or disadvantage: } \\
\text { A11. } 2\end{array}$ & \multicolumn{2}{|c|}{$\begin{array}{l}\text { Puts the enemy off guard, less predictable; } \\
\text { Doesn't allow the enemy to know where you are striking; } \\
\text { Allows attacking the enemy at the time and place of your } \\
\text { choosing; } \\
\text { Easier to plan with respect to logistics, routing, support, and } \\
\text { coordination; }\end{array}$} \\
\hline
\end{tabular}




\begin{tabular}{|l|l|}
\hline & $\begin{array}{l}\text { Consistent with the principles of war; } \\
\text { Better for moral; } \\
\text { Forces the enemy into defensive posture; } \\
\text { Allows Alliance to execute own game plan. }\end{array}$ \\
\hline $\begin{array}{l}\text { Level of confidence related } \\
\text { to this conclusion: }\end{array}$ & $\begin{array}{l}\text { High - considered to reduced the risk of failure significantly; } \\
\text { Alliance has numerical advantage, which allows them to seize the } \\
\text { initiative at an acceptable risk level. Without a numerical } \\
\text { advantage, given relative capability parity, going offensive early } \\
\text { could squander limited resources with serious long-term effects } \\
\text { on the campaign success. }\end{array}$ \\
\hline $\begin{array}{l}\text { Under which evaluation } \\
\text { criteria can you classify this } \\
\text { advantage/disadvantage? }\end{array}$ & $\begin{array}{l}\text { Stealth/Surprise, Concentration, Offensive Action, Surprise, } \\
\text { E11.4 Canomy of Effort, Concentration of Forces, Operational } \\
\text { Capability, Support Capability, Operational Tempo, Operational }\end{array}$ \\
\hline
\end{tabular}

Figure 8. Sample of a structured critiquing text of the Titanium Overcast COA

Here is a brief overview of the method that we followed during this case study carried out with our colleagues at the Defence Research Center, RDDC Valcartier, Canada. We first analyzed the corpus. We observed that the template used to structure the presentation of critics played an important role on the way the arguments have been presented by the specialists. Then, using the comparison framework of argumentation models (presented in the previous sections), we selected two argumentation models which seemed more appropriate to model the arguments found in the corpus: Simari and Loui's model (1992) and Toulmin's model (1958). In section 6.1 we discuss why these two models have been selected.

Then, we modeled a sample of the corpus with both models and found out that Toulmin's model was the most appropriate to formalize the contents of this corpus. However, we noticed that Toulmin's model needs some improvements to model all the relevant elements in the texts. We proposed some adaptations to this model and used the new model to formalize the whole corpus.

\subsection{Selection of Argumentation Models}

Generally, the argumentation models presented and discussed in this paper provide some techniques for knowledge representation. In order to represent argumentative knowledge, two model classes have been distinguished: dialogical models and monological models. 
The dialogical models highlight the dialogical structure of arguments. As illustrated in Table 4, the models proposed by Dung and by Rescher do not support the argumentation process dynamics. Because this dynamics is fundamental in the evaluation and critique of courses of actions, these models are not suitable. In addition, the abstract argumentation system proposed by Lin and Shoham cannot be used to represent argumentative knowledge of our corpus because they are defined in an abstract way without specifying the defense and attack relation between arguments. Finally, the models proposed by Hamblin and MacKenzie, by Amgoud, by Bentahar et al., and by Simari and Loui can be used to represent knowledge used in the critique of courses of actions because they support the different argumentation notions (attack, defense, etc.) and the argumentation dynamics. Particularly, the model proposed by Simari and Loui seems to be more expressive because it uses dialectical trees which illustrate the relations between different propositions.

Rhetorical models proposed by Olbrechts-Tyteca, by Grasso, by Pasquier et al., and by Cabrol-Hatimi offer a technique enabling knowledge representation used in natural argumentation on the basis of rhetorical schemata. The audience's perception is the fundamental characteristic of such models. Consequently, they are not suitable to represent knowledge in cases in which this perception is implicit or not considered. Monological models also provide advanced techniques for knowledge representation. These models emphasize the structure of the arguments by modeling the relationship that can exist between the different components of a given argument. Particularly, Toulmin's model and Reed and Walton's model take into account the knowledge representation in their formulations. The models proposed by Anscombre and Ducrot, and by Breton do not support the argumentation process. Thus, these models are not suitable to represent the argumentative knowledge of our corpus. The model proposed by Toulmin is more expressive than the other models in the sense that the former describes not only premises and conclusions but also warrants which justify the inference of the conclusion from the premises, backing which is the ground underlying the reason, and rebuttal which is the defeater of the claim. 
Hence, in order to model the arguments found in the corpus we selected two models: Simari and Loui's model (1992) and Toulmin's model (1958). Our main conclusions are presented in Section 6.2.

\subsection{Assessment of Simari and Loui's model and Toulmin's model}

Simari and Loui's model allows us to represent several kinds of knowledge describing a COA using the dialectical tree structure and the defense and attack relations between arguments. The argumentation process is illustrated by this structure. However, this model only distinguishes premises and conclusions and it does not illustrate how these conclusions can be inferred from these premises. For example, we cannot represent the description of the Titanium Overcast COA (Section A11.1 in Figure 8) from which we can infer that "seizing initiative" is an advantage. In addition, this model does not provide a representation of explanations. Thus, the only way to take these explanations into account would be to consider them as premises supporting a conclusion. For example, the explanation of why a description is considered as an advantage (Section A11.1 in Figure 8) would be modeled as an argument supporting the fact that "seizing the initiative" is an advantage. However, this way of modeling does not reflect precisely what is described in the scenario. For this reason, the model proposed by Toulmin seems to be more suitable.

An advantage of Toulmin's model is that it takes into account the different components of an argument structure and the links between these components. In addition, it allows us to model the inference rules that are used to infer a conclusion from a set of premises, and it facilitates the construction of textual arguments. However, this model does not illustrate how a warrant supports the inference of a claim from a given data. For example, we cannot explain why "puts the enemy off guard" is a warrant of the fact that "seizing the initiative" is an advantage (Section A11.2 in Figure 8). In addition, this model does not include a justification of rebuttals. For example, we cannot justify why "the lack of operational capability" is a rebuttal of the fact that "seizing initiative" is an advantage. Therefore, to represent such a justification, we need a new model in which the rebuttal becomes the data supporting the negation of the claim. 
Toulmin's model has another limit because rebuttals are considered as counterarguments that cannot be defeated. Consequently, the only way to represent these counterarguments is to consider them as rebuttals of certain claims in a new model. For these reasons we proposed the following improvements to Toulmin's model:

1- Add the names of the argumentation relations on arrows. These names allow us to represent the semantics of the relations between different components.

2- Add a component explaining the warrant. This component explains why the warrant supports the inference of claim from the data.

3- Add a component justifying the rebuttal, and a component that can be used to attack this justification.

These enhancements, as illustrated in Figures 9.1 and 9.2, represent an enriched Toulmin's argument structure for knowledge acquisition. These two figures describe the argumentative structure of a part of the corpus. Indications A11.1 and A11.2 in these figures refer to sections A11.1 and A11.2 in Figure 8. Using this kind of structure, we analyzed the whole corpus and got satisfying results. Since the goal of this section is not to describe in detail the solution proposed in this case study, we will not discuss this model in more details. Rather, our goal is to illustrate how the comparison framework presented in this paper may be practically used. 
Titanium overcast COA is designed to take the offensive as soon as possible after the commencement of hostilities. Phase 2 moves air forces into RL and WL airspace in order to gain air superiority and force RL and WL air forces to be reactive.

(Data. A11.1)

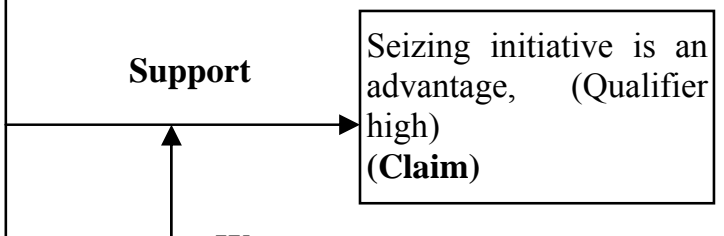

Warrant

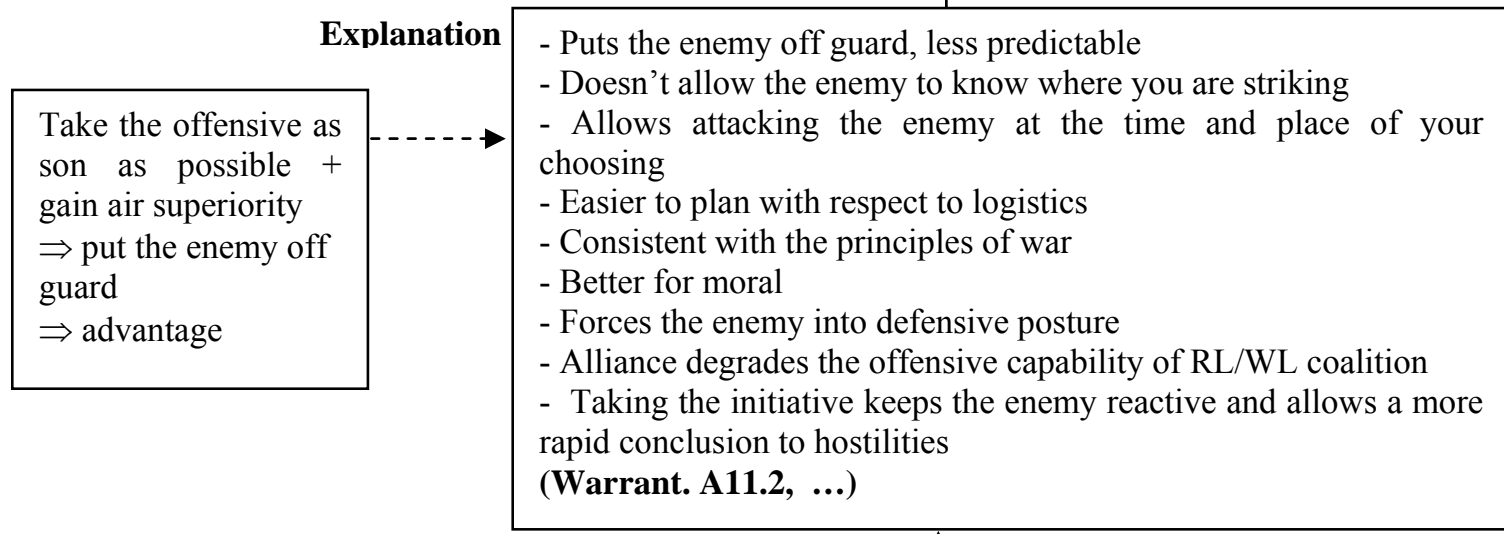

\section{Baking}

- List of principles of war

- Strategic guidance

-Lessons learned and past experiences

(Baking. A11. 5, ...)

Figure 9.1. The enhanced Toulmin's argument structure (part1) 


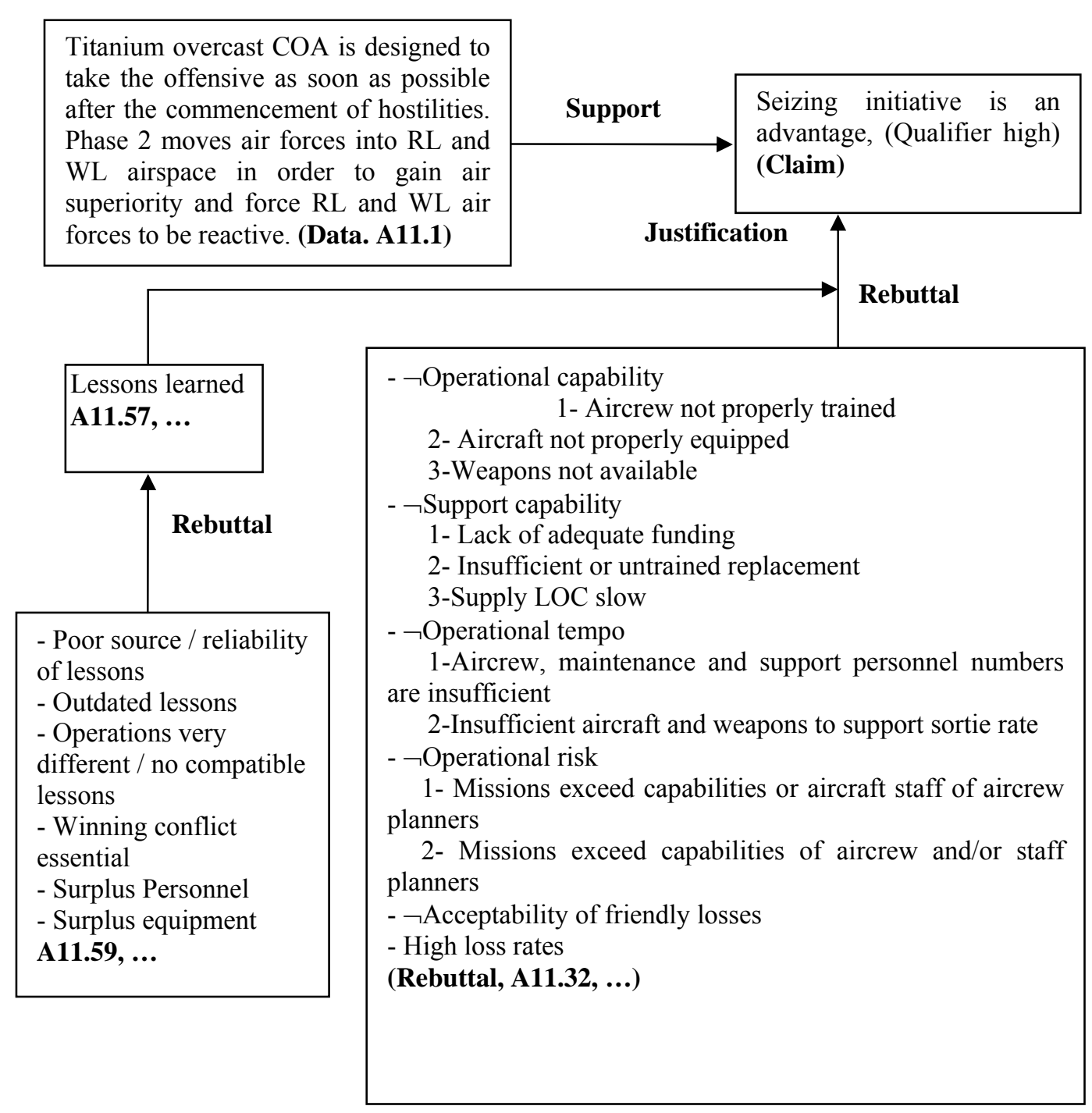

Figure 9.2. The enhanced Toulmin's argument structure (part2)

\section{Conclusion}

In this paper we reviewed some relevant argumentation models presented in the literature and proposed a framework to facilitate their comparison. We distinguished three main categories of models: dialogical models, rhetorical models, and monological models. The main advantage of the dialogical models lies in the fact that they reflect the argumentative reasoning. What is important in these models is not the relevance of a given argument, but the argumentation process through which the participants justify, attack or accept the different exchanged arguments. The main idea behind these models is that the arguments used in the different domains such as law and politics are not used in 
an isolated way but in a process of persuasion and negotiation. Thus, these models aim at representing this process and at studying the relationships between the different arguments. Generally, the argumentation process is represented by a dialogue tree jointly constructed by the two participants (called players). This tree is a finite set of nodes and edges in which each branch is an argument. The final objective is to determine when a player wins an argument dialogue. We can speak here about macro-approaches in which arguments are represented by a set of defeasible or strict rules.

Rhetorical models deal with arguments which are based on the audience's perception of the world, and with evaluative judgments rather than with the goal of establishing the truth of a proposition. The emphasis is put on the audience rather than on the argument itself. For example, in a persuasive dialogue, the rhetorician appeals to the audience's set of beliefs in order to try to persuade this audience, rather than to achieve general acceptability.

Unlike the two other approaches, monological models can be thought of as microapproaches. They focus on the structure of the argument itself and not on the way of using it in order to defend or attack other arguments. Usually, these models are based on informal languages. Their main objective is to represent an argument by a diagram illustrating the different parts of an argument and their relationships. Therefore, these approaches can only be used for evaluation purposes. Particularly, the argumentation schemes proposed by Reed and Walton seem to provide an interesting formalism in the sense that they take into account the micro-structure and the macro-structure of arguments (arguments considered as product and as process). However, determining all possible argument schemes that can be used in natural argumentation, or just classifying them, is not a simple task.

We also illustrated how we used this framework during a case study carried out with colleagues from the Canadian National Defence in order to select an argumentation model for knowledge acquisition and representation, namely to represent knowledge contained in texts critiquing military courses of action. 
We hope that this conceptual framework will be useful to other teams for various research purposes. Furthermore, we hope that other researchers will join us in order to enrich and improve this comparison framework.

\section{Acknowledgements}

This work has been carried out with the partial support of the Canadian National Defence (contract W7701-043300/B, 2005). The first author is also supported by Natural Sciences and Engineering Research Council of Canada (NSERC), Fonds québécois de la recherche sur la nature et les technologies (NATEQ), and Fonds québécois de la recherche sur la société et la culture (FQRSC). We would also like to thank the anonymous reviewers for their valuable and extremely helpful comments and suggestions.

\section{References}

Alvarado, S., and Dyer, M. (1985) Analogy recognition and comprehension in editorials. In Proceedings of the 7th Annual Conference of the Cognitive Science Society, pp. 228235.

Amgoud, L., and Cayrol, C. (2000) A reasoning model based on the production of acceptable arguments, In Linköping Series of Articles in Computer and Information Science, vol. 5 (http://www.ida.liu.se/ext/epa/cis/ufn-00/01/tcover.html).

Amgoud, L., and Hameurlain, N. (2007) An argumentation-based approach for dialogue move selection. In N. Maudet, S. Parsons, I. Rahwan editors, Argumentation in MultiAgent Systems, vol. 4766 of Lecture Notes in Artificial Intelligence, Springer, pp. 128141.

Amgoud, L., Maudet, N., and Parsons, S. (2000a) Arguments, dialogue, and negotiation. In Proceedings of the $14^{\text {th }}$ European Conference On Artificial Intelligence, Germany, pp. 338-342. 
Amgoud, L., Maudet, N., and Parsons, S. (2000b) Modelling dialogues using argumentation. In Proceedings of the $4^{\text {th }}$ International Conference On Multi-Agent Systems, pp. 31-38.

Anscombre, J-C. (1995) Théorie des topoï, Paris: Kimé.

Anscombre, J-C., and Ducrot, O. (1983) L'argumentation dans la langue, Bruxelles: Magrada.

Ashley, K.D., and Rissland, E.L. (2003) Law, learning and representation. Artificial Intelligence, vol. 150, pp. 17-58.

Atkinson, K., Bench-Capon, T.M., and McBurney, P. (2005) A dialogue game protocol for multi-agent argument over proposals for action. Journal of AAMAS, Special issue on Argumentation in Multi-Agent Systems, vol. 11(2), pp. 153-171.

Atkinson, K., Bench-Capon, T.M., and McBurney, P. (2006): Computational representation of practical argument. Knowledge, Rationality and Action, a special section of Synthese, vol. 152(2), pp. 157-206.

Augusto, J.C. (1998) Defeasible Temporal Reasoning. Ph.D. Thesis, Univ. Nacional del Sur, Argentina. Available at: http://www.soi.city.ac.uk/homes/msch

Baroni, P., Giacomin, M., and Guida, G. (2000) Extending abstract argumentation systems theory. In Artificial Intelligence, vol.120(2), pp. 251-270.

Bench-Capon, T.M. (1989) Deep models, normative reasoning and legal expert systems. In Proceedings of the 2nd International Conference on AI and Law, pp. 37-45.

Bench-Capon, T.M. (2003) Persuasion in practical argument using value-based argumentation frameworks. Journal of Logic and Computation, vol.13(3), pp. 429-448.

Bench-Capon, T.M., and Prakken, H. (2006) Argumentation. In A.R. Lodder and A. Oskamp, editors, Information Technology and Lawyers: Advanced Technology in the Legal Domain, from Challenges to Daily Routine, Springer, pp. 61-80. 
Bench-Capon, T.M., Coenen, F., and Orton, P. (1993) Argument-based explanation of the British Nationality Act as a logic program, Computers, Law and AI, vol. 2(1), pp. 53-66.

Bentahar, J. (2005) A unified framework for the pragmatics and semantics of agent communication. Ph.D. Thesis, Laval University, Québec, Canada.

Bentahar, J. (2010) An agent communication protocol for resolving conflicts. In Proceedings of 9th International Joint Conference on Autonomous Agents and Multi Agent Systems, IFAAMAS Press (in press).

Bentahar, J., and Labban, J. (2009) An argumentation-driven model for flexible and efficient persuasive negotiation. In Group Decision and Negotiation Journal, Springer. DOI: 10.1007/s10726-009-9163-0 (online first).

Bentahar, J., Maamar, Z., Benslimane, D., and Thiran, P. (2007a) An argumentation framework for communities of web services. In IEEE Intelligent Systems, vol.22(6), pp. $75-83$.

Bentahar, J., Mbarki, M., and Moulin, B. (2007b) Specification and complexity of strategic-based reasoning using argumentation. In N. Maudet, S. Parsons, I. Rahwan editors, Argumentation in Multi-Agent Systems, vol. 4766 of Lecture Notes in Artificial Intelligence, Springer, pp. 142-160.

Bentahar, J., Mbarki, M., and Moulin, B. (2009a) Strategic agent communication: an argumentation-driven approach. In M. Baldoni, T.C. Son, M.B. van Riemsdijk, and M. Winikoff, editors, Declarative Agent Languages and Technologies VI, vol. 5397 of Lecture Notes in Artificial Intelligence, Springer, pp. 233-250.

Bentahar, J., Meyer, J-J.Ch., and Wan, W. (2009b) Model checking communicative agent-based systems. In Knowledge-Based Systems, Special Issue on Intelligent Software Design, vol. 22(3), pp. 142-159, Elsevier.

Bentahar, J., Moulin, B., and Chaib-draa, B. (2004a) Commitment and argument network: a new formalism for agent communication. In F. Dignum, editor, Advances in Agent Communication, vol. 2922 of Lecture Notes in Artificial Intelligence, Springer, pp. 146-165. 
Bentahar, J., Moulin, B., Meyer, J-J.Ch., and Chaib-draa, B. (2004b) A computational model for conversation policies for agent communication. In Proceedings of the Fifth International Workshop on Computational Logic in Multi-Agent Systems (CLIMA V), vol. 3487 of Lecture Notes in Artificial Intelligence, Springer, pp. 178-195.

Bentahar, J., Moulin, B., Meyer, J-J.Ch., and Chaib-draa, B. (2004c) A logical model for commitment and argument network for agent communication. In Proceedings of $3 \mathrm{rd}$ International Joint Conference on Autonomous Agents and Multi Agent Systems, ACM Press, pp. 792-799.

Bentahar, J., Moulin, B., Meyer, J-J.Ch., and Lespérance, Y. (2007c) A new logical semantics for agent communication. In Proceedings of the Seventh International Workshop on Computational Logic in Multi-Agent Systems (CLIMA VII), vol. 4371 of Lecture Notes in Artificial Intelligence, Springer, pp. 151-170.

Besnard, P., and Hunter, A. (2001) A logic-based theory of deductive arguments. Artificial Intelligence, vol. 128(1-2), pp. 203- 235.

Birnbaum, L. (1982) Argument molecules: a functional representation of argument structures. In American Association for Artificial Intelligence, pp. 63-65.

Bodanza, G.A., and Simari, G.R. (1995) Argumentacion on rebatible con bases disyuntivas. In Proceedings of the Congreso Argentino en Ciencias de la Computation, pp. 313-324.

Breton, P. (1996). L'argumentation dans la communication. Collection Repères, La Découverte, Paris.

Brninghaus, S., and Ashley, K.D. (2003) Predicting the outcome of case-based legal arguments. In G. Sartor, editor, Proceedings of the 9th International Conference on Artificial Intelligence and Law (ICAIL), pp. 233-242.

Cabrol-Hatimi C. (1999) Un Modèle de formalisation des argumentations naturelles basé sur la notion de force persuasive : application à la planification des idées. Thèse de Doctorat,Université de Toulouse 1, France. 
Cabrol-Hatimi, C., and Tazi, S. (2000) APLA : A human-machine cooperative system for arguments selection support. In Proceedings of COOP'2000.

Chesnevar, C.I. (1996) El Problema de la inferencia en sistemas argumentativos: alternativas para su solucion. M.Sc. Thesis. University Nacional del Sur.

Chesnevar, C.I., and Simari, G.R. (1998) Formalization of defeasible argumentation using labelled deductive systems. In Proceedings of the IV Congreso Argentino en Ciencias de la Computacion. Univ. Nacional del Comahue, pp. 1247-1259.

Chesnevar, C.I., Maguitman, A.G., and Loui R.P. (2000) Logical models of argument, ACM Computing Surveys, vol. 32(4), pp. 337-383.

Chesnevar, C.I., McGinnis, J., Modgil, S., Rahwan, I., Reed, C., Simari, G., South, M., Vreeswijk, G., and Willmott, S. (2006). Towards an Argument Interchange Format. The Knowledge Engineering Review, vol. 21(4), pp. 293-316.

Clark, P. (1991) A Model of Argumentation and its Application in a Cooperative Expert System. Ph.D. Thesis, Turing Institute, University of Strathclyde, Glasgow, U.K.

Coombs, M., and Alty, J. (1984) Expert systems: an alternative paradigm. International Journal of Man-Machine Studies, vol. 20, pp. 21-43.

Delrieux, C. (1995) Incorporando razonamiento plausible en los sistemas de razonamiento revisable. M.Sc. Thesis. Univ. Nacional del Sur.

Dieng, R. (1989) Generation of Topoi from expert systems. CCAI 6:4, P.Y.Raccah (Edt.), Gand.

Doyle, J. (1979) A Truth Maintenance System. Artificial Intelligence, vol. 12, pp. 231272.

Ducrot, O. (1991) Dire ou ne pas Dire, Principes de sémantique linguistique, Herman, Collection Savoir, Paris. 
Dung, P.M. (1995) On the acceptability of arguments and its fundamental role in nonmonotonic reasoning, logic programming and n-person games. Artificial Intelligence, vol. 77, pp. 321-357.

Falappa, M.A. (1999). Teorìa de cambio de creencias y sus aplicaciones sobre bases de conocimiento. Ph.D. Thesis, Dept. de Cs. de la Computacìon. Univ. Nacional del Sur.

Farley, A., and Freeman, K. (1995) Toward formalizing dialectical argumentation. In Proceedings of the 3rd. International Conference of the Society for the Study of Argumentation, pp. 156-165.

Flowers, M., McGuire, R., and Birnbaum, L. (1982) Adversary arguments and the logic of personal attacks. In Fetzer, J.H, Strategies for Natural Language Processing, pp. 275294.

Fox, J., and Das, S. (2000) Safe and sound. Artificial Intelligence in Hazardous Applications, AAAI Press, The MIT Press.

Fox, J., Krause, P., and Elvan-Goransson, M. (1993) Argumentation as a general framework for uncertain reasoning. In Proceedings of the 9th Conference on Uncertainty in AI, Morgan-Kaufmann, pp. 428-434.

Freeman, J.B. (1991) Dialectics and the Macrostructure of Arguments. Foris.

Galarreta, D., and Trousse, B. (1996) Place de l'argumentation dans la conception d'outils d'assistance à une activité de résolution de problème. In (Raccah 1996), pp. 79-103.

Garcìa, A.J., and Simari, G.R. (2004) Defeasible logic programming: an argumentative approach. Theory and Practice of Logic Programming, vol. 4(1), pp. 95-138.

Glasspool, D.W., Fox, J., Castillo, F.D., and Monaghan, V. (2003) Interactive decision support for medical planing. In Proceedings of Artificial Intelligence in Medicine, 9th Conference on Artificial Intelligence in Medicine in Europe (AIME 2003), vol. 2780 of Lecture Notes in Computer Science, Springer, pp. 335-339.

Gordon, T.F. (1994) Computational dialectics. In First Workshop on Computational Dialectics. Twelfth National Conference on Artificial Intelligence-AAAI '94. 
Gordon, T.F., Prakken, H., and Walton, D. (2007). The carneades model of argument and burden of proof. Artificial Intelligence, vol. 171, pp: 875-896.

Grasso, F. (2002) Towards a framework for rhetorical argumentation. In Proceedings of the $6^{\text {th }}$ Workshop on the Semantics and Pragmatics of Dialogue (EDILOG'02), UK, pp. 53-60.

Grasso, F. (2003) A mental model for a rhetorical arguer. In Proceedings of the European Cognitive Science Society Conference. Schmalhofer, F., Young, R., and Katz, G. (eds.), LEA, Germany.

Greenwood, K., Bench-Capon, T., and McBurney, P. (2003) Structuring dialogue between the People and their representatives. In R. Traunmuller, editor, Electronic Government: Proceedings of the Second International Conference (EGOV03), Czech Republic, Lecture Notes in Computer Science 2739, Springer, pp. 55-62.

Groarke, L., Tindale, C., and Fisher, L. (1997) Good Reasoning Matters! Oxford University Press, Toronto.

Grosz, B.J., and Sidner, C.L. (1986) Attention, intentions and the structure of Discourse. Computational Linguistics, vol. 12(3), pp. 175-204.

Habermas, J. The theory of Communicative Action. Volume 1 and 2, Polity Press, Cambredge, UK, 1984.

Hamblin, C.L. (1999) Fallacies. Methuen, 1970.

Hollnagel E. (1987) Commentary: Issues in Knowledge-based Decision Support, International Journal of Man-Machine Studies, vol. 27, pp. 743-751.

Huhns M.N., and Bridgeland D. M. (1991) Multiagent truth maintenance, IEEE Transactions on Systems, Man, and Cybernetics, SMC-21, vol. 6, pp. 1437-1445.

Johnson, R. (2000) Manifest Rationality. Lawrence Erlbaum.

Kakas, A.C., Miller, R., and Toni, F. (1999) An argumentation framework for reasoning about actions and change. In Proceedings of LPNMR 99, LNCS 1730, pp. 78-91. 
Karacapilidis, N., and Papadias, D. (2001) Computer supported argumentation and collaborative decision making: The hermes system. In Information Systems, vol. 26(4), pp. 259-277.

Karsenty, L., and Brézillon, P.J. (1995) Cooperative Problem Solving and Explanation. Expert Systems with Applications, vol. 8(4), pp. 445-462.

Kasper, G.M. (1996) A theory of decision support system design for user calibration. Information Systems Research, vol. 7(2), pp. 215-232.

Konolige, K., and Pollack, M. (1989) Ascribing plans to agents. In Proceedings of the International Joint Conference on Artificial Intelligence, USA.

Konolige, K., and Pollack, M. (1993) A representational theory of intention. In Proceedings of the 13th International Joint Conference in Artificial Intelligence (IJCAI), France.

Kraus, P., Ambler, S., Elvang-Goransson, M., and Fox, J. (1995) A logic of argumentation for reasoning under uncertainty, Computational Intelligence, vol. 11(1), pp. 113-131.

Langlotz, C.P., and Shortliffe, E.H. (1983) Adapting a consultation system to critique user plans. International Journal of Man-Machine Studies, vol. 19, pp. 479-496.

Lin F., and Shoham Y. (1989) Argument systems: a uniform basis for nonmonotonic reasoning. In Proceedings of First International Conference on Knowledge Representation and Reasoning, Toronto, pp. 245-255.

Lodder, A.R. (1997) On structure and naturalness in dialogical models of argumentation. In J.C. Hage et al. (eds.), Legal Knowledge-Based Systems. JURIX: The eleventh Conference, GNI, Nijmegen, pp. 45-58.

Lodder, A.R. (1998) Procedural arguments. In A. Oskamp et al. (eds.), Legal KnowledgeBased Systems. JURIX: The tenth Conference, GNI, Nijmegen, pp. 21-32.

Lodder, A.R. (1999). DiaLaw - On Legal Justification and Dialogical Models of Argumentation. Dordrecht, Kluwer Academic Publishers. 
Loui, R.P. (1987) Defeat among arguments: a system of defeasible inference, Computational Intelligence, vol. 3, pp. 157-365.

Loui, R.P., Norman J. (1995) Rationales and argument moves, Artificial Intelligence and Law, vol. 3 (3), pp. 159-189.

Loui, R.P., Norman, J., Alteper, J., Pinckard, D., Craven, D., Lindsay, J., and Foltz, M. (1997) Progress on Room 5. A testbed for public interactive semi-formal legal argumentation. In Proceedings of the Sixth International Conference on Artificial Intelligence and Law, New York: ACM, pp. 207-214.

MacKenzie, J. (1979) Question-begging in non-cumulative systems. Journal Of Philosophical Logic, vol. 8, pp. 117-133.

MacKenzie, J. (1981) The dialectics of logic. Logique et Analyse, vol. 94, pp. 159-177.

Miller, P.L. (1984) A Critique Approach to Expert Computer Advice : ATTENDING, Pitman.

Moeshler, J. (1985) Argumentation et conversation: Eléments pour une analyse pragmatique du discours, Paris: Hatier.

Mommers, L. (2002) Applied legal epistemology. Ph.D. Thesis. Leiden University. The Netherlands.

Moulin, B., Irandoust, H., Bélanger, M., and Desbordes, G. (2002) Explanation and Argumentation Capabilities : Towards the creation of more persuasive agents. Artificial Intelligence Review, vol. 17, pp. 169-222.

O'Keefe, D. (1977) Two concepts of argument. Journal of the American Forensic Society, vol. 13, pp. 121-128.

O'Keefe, D. J. (1990) Persuasion: Theory and Research, SAGE Publications.

Parsons, S., and Jennings, N.R. (1996) Negotiation through argumentation-a preliminary report. In Proceedings of the $2^{\text {nd }}$ International Conference On Multi Agent Systems, pp. 267-274. 
Parsons, S., Sierra, and C., Jennings N. (1998) Agents that reason and negotiate by arguing. Journal of Logic and Computation, vol. 8(3), pp. 261-292.

Parsons, S., Wooldridge, M., and Amgoud, L. (2002) An analysis of formal inter-agent

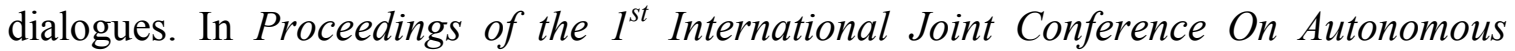
Agents and Multi-Agent Systems (AAMAS 02), Italy, pp. 394-401.

Parsons, S., Wooldridge, M., and Amgoud, L. (2003) On the outcomes of formal interagent dialogues. In Proceedings of the $2^{\text {nd }}$ International Joint Conference On Autonomous Agents and Multi-Agent Systems (AAMAS 03), Australia, pp. 616-623.

Pasquier, P., Rahwan, I., Dignum, F., and Sonenberg, L. (2006) Argumentation and persuasion in the cognitive coherence theory. In P. Dunne and T. Bench-Capon, editors, Proceedings of the 1st International Conference on Computational Models of Argument (COMMA), IOS Press, pp. 223-234.

Perelman, C., and Olbrechts-Tyteca, L. (1969) The New Rhetoric: a Treatise on Argumentation. Notre Dame Press, University of Notre dame.

Pollack, M.E., Hirschberg, J., and Webber, B. (1982) User participation in the reasoning process of expert systems. In Proceedings of First National Conference on Artificial Intelligence, pp. 358-361.

Pollock, J.L. (1974) Knowledge and Justification. Princeton.

Pollock, J.L. (1991) A theory of defeasible reasoning. International Journal of Intelligent Systems, John Willy \&Sons, pp. 33-54.

Pollock, J.L. (1992) How to reason defeasibly?, Artificial Intelligence, vol. 57, pp. 1-42.

Pollock, J.L. (1994) Justification and defeat, Artificial Intelligence, vol. 67, pp. 377-407.

Pollock, J.L. (1995) Cognitive Carpentry: A Blueprint for How to Build a Person. MIT.

Prakken, H., and Sartor, G. (1996) A dialectical model of assessing conflicting arguments in legal reasoning. Artificial Intelligence and Law, vol. 4, pp. 331-368. 
Prakken, H., and Sartor, G. (1997) Argument-based extended logic programming with defeasible priorities, Journal of Applied Non-Classical Logics, vol. 7(1), pp. 25-75.

Prakken, H., and Sartor, G. (1998) Modelling reasoning with precedents in a formal dialogue game. Artificial Intelligence and Law, vol. 6, pp. 231-287.

Prakken, H., and Vreeswijk, G. (2002) Logics for defeasible argumentation. In D.M. Gabby and F. Guenthner, editors, Handbook of Philosophical Logic, Kluwer Academic Publishers, $2^{\text {nd }}$ edition, vol. 4, pp. 219-318.

Raccah, P-Y. (edt.) (1996) Topoï et gestion des connaissances, Paris: Masson.

Rahwan, I., and McBurney, P. (2007) Guest editors' introduction: argumentation technology. In IEEE Intelligent Systems, vol. 22(6), pp. 21-23.

Rahwan, I., Ramchurn, S.D., Jennings, N.R., McBurney, P., Parsons, S., and Sonenberg, L. (2004) Argumentation-based negotiation. Knowledge Engineering Review, pp. 343375.

Rahwan, I., Zablith, F., and Reed, C. (2007) Laying the foundations for a world wide argument web. Artificial Intelligence, vol. 171(10-15), pp. 897-921.

Ramchurn, S.D., Sierra, C., Godo, L., and Jennings, N.R. (2007) Negotiating using rewards. In Artificial Intelligence, vol. 171(10-15), pp. 805-837.

Reed, C., and Norman, T.J. (2003) A roadmap of research in argument and computation. In Argumentation Machines - New Frontiers in Argument and Computation, C. Reed and T.J. Norman, editors. Kluwer Academic Publishers, pp. 1-13.

Reed, C., and Rowe, G. (2001) Araucaria: Software for puzzles in argument diagramming and XML. Technical Report, Department of Applied Computing, University of Dundee, Scotland, UK.

Reed, C., and Walton, D. (2003) Argumentation schemes in argument-as-process and argument-as-product. In Proceedings of the Conference Celebrating Informal Logic@25, Windsor, Ontario. 
Rescher, N. (1977) Dialectics, a Controversy-Oriented Approach to the Theory of Knowledge. State University of New York Press, USA.

Rissland, E., Skalak D., and Friedman, M. (1993) Bankxx: A program to generate argument through case-based search. In Proceedings of the Fourth International Conference on AI an Law, Amsterdam, the Netherlands, pp. 117-124.

Ryan, E. (1992) Aristotle and the tradition of rhetorical argumentation. In Argumentation Journal, vol. 6(3), pp. 291-296.

Schroeder, M. (1999) An Efficient Argumentation Framework for Negotiating Autonomous Agents, In Proceedings of the $9^{\text {th }}$ European Workshop on Modelling Autonomous Agents in a Multi-Agent World (MAAMAW'99), Valencia, Spain, pp. 140149.

Schroeder, M. (2000) Towards a Visualization of Arguing Agents, To appear in Journal of Future Generation Computing systems, Elsevier.

Sierra, C., Jennings, N.R., Noriega, P., and Parsons, S. (1998) A framework for argumentation-based negotiation. In Intelligent agents IV, Singh, M.P, Rao, A., Wooldridge, M. (eds.), LNAI 1365, Springer, pp. 177-192.

Sillince, J.A.A. (1994) Multi-agent conflict resolution: a computational framework for an intelligent argumentation program, Knowledge-Based Systems, vol. 7(2), pp. 75-90.

Silverman, B.G. (1992) Survey of Expert Critiquing Systems: Practical and Theoretical Frontiers. Communications of the ACM, vol. 35 (4), pp. 106-127.

Simari, G.R. (1989) A Mathematical Treatment of Defeasible Reasoning and its Implementation. Ph.D. Thesis, Washington University. USA.

Simari, G.R., and Garcìa, A.J. (1995) A knowledge representation language for defeasible argumentation. In CLEI'95, Canela, Brasil, pp. 661-672.

Simari, G.R., Chesnevar, C.I., and Garcìa, A.J. (1994) The role of dialectics in defeasible argumentation. In Anales de la XIV Conferencia Internacional de la Sociedad Chilena para Ciencias de la Computacion. Univ. de Concepcìn, Chile, pp. 270-281. 
Simari, G.R., Loui R.P. (1992) A mathematical treatment of defeasible reasoning and its implementation, Artificial Intelligence, vol. 53, pp. 125-157.

Stranieri, A., and Zeleznikow, J. (1999) A survey of argumentation structures for intelligent decision support. In Proceedings of Fifth International Conference of the International Society for Decision Support Systems.

Sycara, K.P. (1990) Persuasive argumentation in negotiation. Theory and Decision, vol. 28, pp. 203-242.

Tindale, C. (1999). Acts of Arguing, A Rhetorical Model of Argument. State University Press of New York, Albany, NY, USA.

Tohmé, F. (1997) Negotiation and defeasible reasons for choice. In Proceedings of the Stanford Spring Symposium On Qualitative Preferences in Deliberation and Practical Reasoning, pp. 95-102.

Toulmin, S. (1958) The Uses of Argument, Cambridge University Press, Cambridge, England.

Vahidov, R., and Elrod, R. (1999) Incorporating critique and argumentation in DSS. Decision Support Systems, vol. 26, Elsevier, pp. 249-258.

Verheij, B. (1996) Rules, reasons and arguments: formal studies of argumentation and defeat, Ph.D. Thesis, Maastricht University, Maastricht (Netherlands).

Verheij, B. (1998) Argue! An implemented system for computer-mediated defeasible argumentation, In proceedings of the Tenth Netherlands/Belgium Conference on Artificial Intelligence, Amsterdam: CWI, pp. 57-66.

Vreeswijk, G.A. (1993) Studies in defeasible argumentation. Ph.D. Thesis, Vrije University, Holland.

Vreeswijk, G.A. (1995) IACAS: an implementation of Chisholm's principles of knowledge, In Proceedings of the Second Dutch/German Workshop on Nonmonotonic reasoning, Delft University of Technology, pp. 225-234. 
Vreeswijk, G.A. (1997) Abstract argumentation systems, Artificial Intelligence, vol. 90(1-2), pp. 225-279.

Walton, D.N. (1996) Argument Structure: A Pragmatic Theory, University of Toronto Press.

Walton, D.N., and Krabbe, E.C.W. (1995) Commitment in Dialogue: Basic Concepts of Interpersonal Reasoning, State University of New York Press, Albany N.Y.

Willmott, S., Vreeswijk, G., South, M., Chesnevar, C., Simari, G., McGinis, J., Rahwan, I., Reed, C., and Modgil, S. (2006) Towards an argument interchange format for multiagent systems. In Proceedings of the International Workshop on Argumentation in Multi-Agent Systems, vol. 4766 of Lecture Notes in Artificial Intelligence, Springer, pp. 17-34.

Ye, L.R. (1995) The value of explanation in expert systems for auditing: an experimental investigation. Expert Systems with Applications, vol. 9(4), pp. 543-556. 